\title{
BAYESIAN LATENT HIERARCHICAL MODEL FOR TRANSCRIPTOMIC META-ANALYSIS TO DETECT BIOMARKERS WITH CLUSTERED META-PATTERNS OF DIFFERENTIAL EXPRESSION SIGNALS
}

\author{
By Zhiguang HuO ${ }^{1}$, Chi Song ${ }^{2}$ And GeORGe Tseng ${ }^{1,2}$ \\ University of Florida, Ohio State University and University of Pittsburgh
}

Due to the rapid development of high-throughput experimental techniques and fast-dropping prices, many transcriptomic datasets have been generated and accumulated in the public domain. Meta-analysis combining multiple transcriptomic studies can increase the statistical power to detect disease-related biomarkers. In this paper we introduce a Bayesian latent hierarchical model to perform transcriptomic meta-analysis. This method is capable of detecting genes that are differentially expressed (DE) in only a subset of the combined studies, and the latent variables help quantify homogeneous and heterogeneous differential expression signals across studies. A tight clustering algorithm is applied to detected biomarkers to capture differential meta-patterns that are informative to guide further biological investigation. Simulations and three examples, including a microarray dataset from metabolism-related knockout mice, an RNA-seq dataset from HIV transgenic rats and cross-platform datasets from human breast cancer are used to demonstrate the performance of the proposed method.

1. Introduction. With the rapid development of high-throughput experimental techniques and fast-dropping prices, many transcriptomic datasets have been generated and deposited into public databases. In general each dataset contains a small to moderate sample size which requires caution in gauging the accuracy and reproducibility of detected biomarkers [Domany (2014), Simon (2005), Simon et al. (2003)]. Meta-analysis combining multiple transcriptomic studies can increase statistical power and provide robust conclusions from various platforms and sample cohorts [Ramasamy et al. (2008)]. Tseng, Ghosh and Feingold (2012) presented a comprehensive review of methods and applications in the microarray meta-analysis field and categorized existing methods into combining $p$-values, combining effect sizes, direct merging (aka mega-analysis) and combining nonparametric ranks. In general meta-analysis can be viewed as two-step information reduction and combination tools for adjusting batch effects (such as different experimental platforms, protocols and bias) across studies to draw a more efficient

\footnotetext{
Received September 2016; revised February 2018.

${ }^{1}$ Supported by NIH RO1CA190766.

${ }^{2}$ To whom correspondence should be addressed.

Key words and phrases. Transcriptomic differential analysis, meta-analysis, Bayesian hierarchical model, Dirichlet process.
} 
and accurate conclusion. This paper focuses on combining $p$-value methods, and we will discuss other potential approaches of adjusting batch effects and directly merging studies in the discussion section.

Following conventions in Birnbaum (1954) and Li and Tseng (2011), two hypothesis settings have been considered in meta-analysis. In the first setting (namely $\mathrm{HS}_{A}$ ), we aim to detect biomarkers that are differentially expressed (DE) in all studies: $H_{0}: \vec{\theta} \in \bigcap\left\{\theta_{s}=0\right\}$ versus $H_{A}: \vec{\theta} \in \bigcap\left\{\theta_{s} \neq 0\right\}$, where $\theta_{s}$ is the effect size of study $s, 1 \leq s \leq S$. Throughout this manuscript effect size refers to unstandardized effect size [Cooper, Hedges and Valentine (2009)] (difference of group means or unstandardized regression coefficients). In the second setting $\left(\mathrm{HS}_{B}\right)$, targeted biomarkers are DE in one or more studies: $H_{0}: \vec{\theta} \in \bigcap\left\{\theta_{S}=0\right\}$ versus $H_{A}: \vec{\theta} \in \bigcup\left\{\theta_{S} \neq 0\right\}$. In view of overly stringent criterion in $\mathrm{HS}_{A}$ in noisy genomic data and when $S$ is large, Song and Tseng (2014) proposed a robust setting $\mathrm{HS}_{r}$, requiring that $r$ or more studies are DE: $H_{0}: \vec{\theta} \in \bigcap\left\{\theta_{s}=0\right\}$ versus $H_{A}: \vec{\theta} \in \sum \mathbb{I}\left\{\theta_{s} \neq 0\right\} \geq r$, where $\mathbb{I}\{\cdot\}$ is an indicator function taking value one if the statement is true and zero otherwise, and $r$ is usually pre-estimated with $S / 2 \leq r \leq S$. Song and Tseng (2014) also proposed using the $r$ th-ordered $p$-value (rOP, $T^{\mathrm{rOP}}=p_{(r)}$ ) to test $\mathrm{HS}_{r}$. Generally speaking, because $\mathrm{HS}_{A}$ and $\mathrm{HS}_{r}$ are designed to detect consensus biomarkers across the combined studies, they are most biologically interesting. However, when heterogeneous differential expression signals across studies are expected (e.g., studies come from different tissues or brain regions in the two mouse/rat examples in Section 4), biomarkers detected from $\mathrm{HS}_{B}$ can also be of interest. Chang et al. (2013) conducted a comparative study evaluating 12 popular microarray meta-analysis methods targeting the three hypothesis settings $\left(\mathrm{HS}_{A}, \mathrm{HS}_{B}\right.$ and $\left.\mathrm{HS}_{r}\right)$.

Strictly speaking, $\mathrm{HS}_{B}$ is a sound hypothesis setting, and statistical tests for $\mathrm{HS}_{B}$ are easier to develop. Most popular $p$-value aggregation methods, such as Fisher's method [Fisher (1934)] and Stouffer's method [Stouffer et al. (1949)], aim for this $\mathrm{HS}_{B}$ setting. In the literature $\mathrm{HS}_{B}$ is also called a conjunction or intersection hypothesis [Benjamini and Heller (2008)]. On the other hand $\mathrm{HS}_{A}$ is a somewhat defective hypothesis setting in the sense that the null and alternative spaces are not complementary. For example, if we apply the maximum $p$-value test $\left(T^{\operatorname{maxP}}=\max _{s} p_{s}\right.$, where $p_{s}$ is the $p$-value for study $\left.s\right)$ for $\mathrm{HS}_{A}$, and we reasonably assume that $p$-values independently follow $\operatorname{UNIF}(0,1)$ under the null hypothesis, then the null distribution of $T^{\operatorname{maxP}}$ is $\operatorname{Beta}(S, 1)$. This test is anticonservative when there exist genes DE in some but not all of the studies. The problem mainly comes from the noncomplementary null and alternative spaces in $\mathrm{HS}_{A}$ (and also $\mathrm{HS}_{r}$ ). A more appropriate hypothesis setting for the same purpose is $\mathrm{HS}_{\bar{A}} \equiv H_{0}: \vec{\theta} \in \bigcup\left\{\theta_{s}=0\right\}$ versus $H_{A}: \vec{\theta} \in \bigcap\left\{\theta_{S} \neq 0\right\}$ (and $\mathrm{HS}_{\bar{r}} \equiv H_{0}: \sum \mathbb{I}\left\{\theta_{s} \neq 0\right\}<r$ versus $\left.H_{A}: \sum \mathbb{I}\left\{\theta_{s} \neq 0\right\} \geq r\right)$. Benjamini and Heller (2008) proposed a legitimate but conservative test for $\mathrm{HS}_{\bar{A}}$ and $\mathrm{HS}_{\bar{r}}$. In genomic applications the composite null hypotheses of $\mathrm{HS}_{\bar{A}}$ and $\mathrm{HS}_{\bar{r}}$ are complicated by 
the fact that genes can be differentially expressed in up to $S-1$ (for $\mathrm{HS}_{\bar{A}}$ ) or $r-1$ (for $\mathrm{HS}_{\bar{r}}$ ) studies with different levels of effect sizes. Under such a scenario it becomes very difficult to characterize the null distribution for hypothesis tests in a frequentist setting. Theoretically, it is necessary to borrow differential expression information across genes to significantly improve statistical power. Bayesian hierarchical modeling can provide a convenient solution for this purpose. Efron (2008) and Efron (2009) applied empirical Bayes methods to control the false discovery rate (FDR) in single microarray studies. Muralidharan (2010) further extended these works to allow for the simultaneous modeling of both empirical null and alternative distribution of the test statistics. Zhao, Kang and Yu (2014) incorporated pathway information to select genes using the Bayesian mixture model. Despite these successful applications a Bayesian method that combines multiple studies and detects DE genes based on various meta-analysis hypothesis settings is yet to be developed. In this paper we propose a Bayesian latent hierarchical model (BayesMP, named after the Bayesian method for meta-patterns) that uses a nonparametric Bayesian method to effectively combine information across genes for direct testing for $\mathrm{HS}_{\bar{A}}$ (as well as $\mathrm{HS}_{B}$ and $\mathrm{HS}_{\bar{r}}$ ) on a genome-wide scale. In simulations we show successful Bayesian false discovery rate control of BayesMP, while the original maxP and rOP method using a beta null distribution loses FDR control for the $\mathrm{HS}_{\bar{A}}$ and $\mathrm{HS}_{\bar{r}}$ settings.

Traditionally, meta-analysis aims to pool consensus signals to increase statistical power (e.g., by fixed or random effects models). Recently, researchers have recognized the existence of heterogeneous signals among cohorts and the importance of their characterization in meta-analysis. For example, Figure 1(a) shows three modules of detected biomarkers from the RNA-seq HIV transgenic rat data using BayesMP and meta-pattern clustering (see Section 4.2 for details). Modules I and II are consensus biomarkers that are either all down-regulated or all up-regulated across three brain regions. In contrast the biomarkers in module III are downregulated in HIP but up-regulated in PFC and STR. Such biomarkers are somewhat expected because it is well known that different brain regions are responsible for different functions such as reasoning, recognition, visual inspection and memory/speech. Several approaches, such as the adaptive weighting (or subset) method [Li and Tseng (2011), Bhattacharjee et al. (2012)] and lasso variable selection [Li et al. (2014)], have been proposed for quantifying and inferring such heterogeneity. In the adaptively weighted Fisher's method [AW-Fisher; Li and Tseng (2011)], for example, heterogeneity of differential expression signals in each study is categorized by $w_{g s}$ as 0 or 1 weights ( 1 representing differential expression for gene $g$ in study $s$, and 0 for nondifferential expression). Specifically, AW-Fisher considers weighted Fisher's statistics, that is, $T\left(\vec{W}_{g}\right)=-2 \sum_{s=1}^{S} w_{g s} \cdot \log \left(p_{g s}\right)$, where $\vec{W}_{g}=\left(w_{g 1}, \ldots, w_{g S}\right)$ is the vector of 0 or 1 weights reflecting gene-specific heterogeneous contribution of each study, and $p_{g s}$ is the $p$-value of gene $g$ in study $s$ - and adaptively searches the best weight vector for gene $g$ by minimizing the re- 


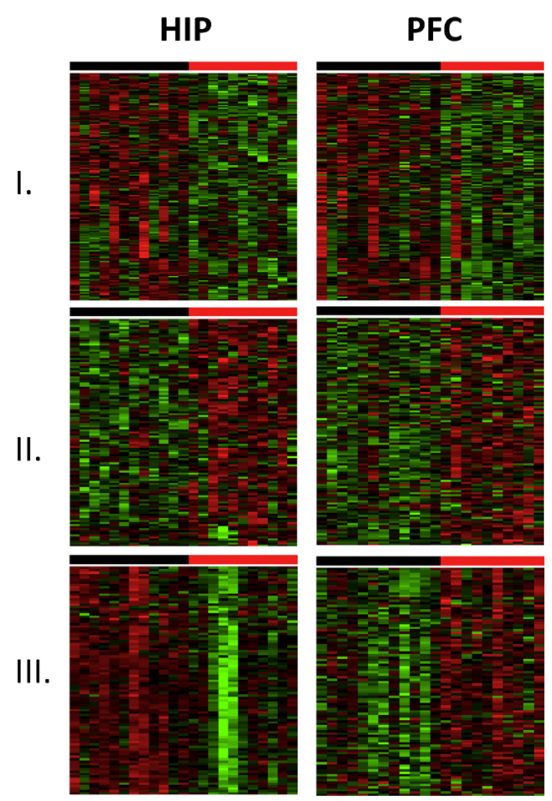

(a) Heatmap

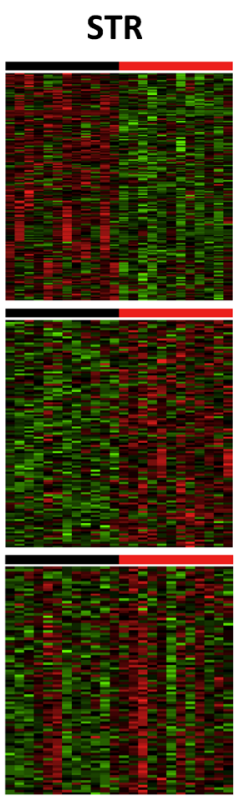

ans

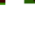

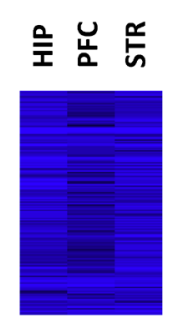
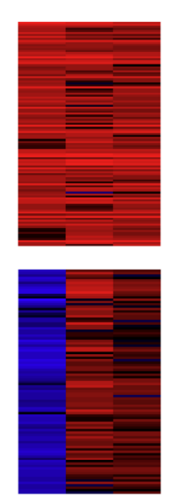

(b) CS
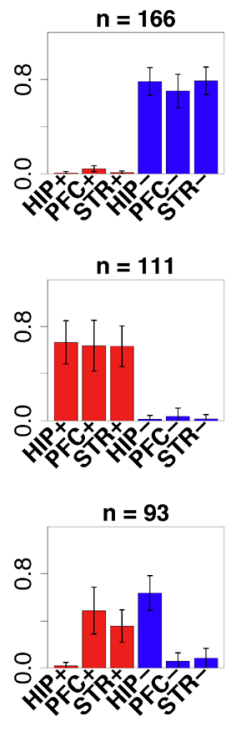

(c) bar plot

FIG. 1. Three meta-pattern modules of biomarkers from HIV transgenic rats example. Each row (module I, II, and III) shows a set of detected biomarkers showing similar meta-pattern of differential signals. (a) Heatmaps of detected genes (on the rows) and samples (on the columns) for each brain region (HIP, PFC, or STR), where each brain region represents a study (i.e., HIP for $s=1$, PFC for $s=2$, STR for $s=3)$. The black color bar on top represents $F 334$ rats (control), and the red color bar on top represents HIV transgenic rats (case). (b) Heatmaps of confidence scores (CS) (genes on the rows and three studies on the columns). The confidence score is described in Section 2.3 which ranges from -1 (blue color for down-regulation) to 1 (red color for up-regulation). (c) Bar plots of mean posterior probability for $Y_{g s}=1$ (red color for up-regulation) and $Y_{g s}=-1$ (blue color for down-regulation) for each module in each brain region. Error bar represents standard deviation across all genes in the module. The number of genes is shown on top of each bar plot.

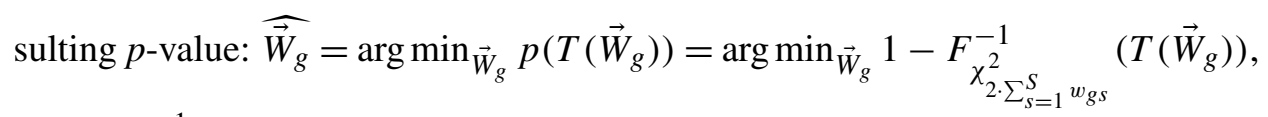
where $F_{\chi_{w}^{2}}^{-1}$ is the inverse CDF of chi-squared distribution with degree of freedom $w$. The 0 or 1 weights estimated from AW-Fisher help cluster detected biomarkers by their differential expression meta-patterns but have a disadvantage of hard thresholding without quantification of variability. When $S$ is large, the number of all possible $2^{S}-1$ weight combinations also makes the problem intractable. In BayesMP the differential expression indicators naturally come with variability estimates from posterior distribution (see a confidence score to be defined later in Section 2.3). In BayesMP we also adopt a cosine dissimilarity measure on these posterior distributions and apply tight clustering [Tseng and Wong (2005)] to identify biomarkers of different meta-patterns (e.g., see the three mod- 
ules of biomarkers in Figure 1). Unsupervised clustering of the expression pattern across studies identifies data-driven modules of biomarkers of different metapatterns and provides interpretable results for further biological investigation. For example, it is interesting to investigate why biomarkers in module III are downregulated in HIP but up-regulated in PFC and STR. We note here that our proposed cluster analysis to categorize detected biomarkers by studying heterogeneity in meta-analysis is a relatively novel concept. It is different from popular practices of clustering genes for identifying coexpression gene modules or clustering samples for discovering disease subtypes [e.g., Huo et al. (2016)].

In this paper Section 2 establishes the methodology, estimation and inference of BayesMP. Section 3 evaluates the performance of the proposed method using simulation datasets. Section 4 shows the application to three real examples. Finally, Section 5 provides conclusions and discussions.

2. Methods. For the ease of discussion we focus on detecting DE genes in two-class comparison in this manuscript. The method can be easily extended for studies with numerical or survival outcomes. In a meta-analysis combining $S$ studies with $G$ genes, we denote $p_{g s}$ as the one-sided $p$-value testing for downregulation for gene $g$ in study $s$, where $1 \leq g \leq G$ and $1 \leq s \leq S$. These $p$-values can be calculated from SAM [Tusher, Tibshirani and Chu (2001)] or limma [Smyth (2005)] for microarray studies (or RNA-seq studies with RPKM data) and edgeR [Robinson, McCarthy and Smyth (2010)] or DEseq [Anders and Huber (2010)] for RNA-seq studies with count data. As a result our model is flexible to mixed studies of different platforms (e.g., microarray or RNA-seq) and study designs (e.g., casecontrol, numerical outcome or survival outcome). Throughout this manuscript we use limma and edgeR to obtain the $p$-values. For modeling convenience we transform the one-sided $p$-values into $Z$-statistics, that is, $Z_{g s}=\Phi^{-1}\left(p_{g s}\right)$, where $\Phi^{-1}(\cdot)$ is the inverse cumulative density function (CDF) of standard Gaussian distribution. $Z_{g s}$ is the input data for BayesMP.

2.1. Bayesian hierarchical mixture model. Denote by $\theta_{g_{s}}$ the effect size of gene $g$ in study $s$ and by $Y_{g s}$ an indicator variable s.t. $Y_{g s}=1$ if $\theta_{g s}>0$ (upregulation), $Y_{g s}=-1$ if $\theta_{g s}<0$ (down-regulation) and $Y_{g s}=0$ if $\theta_{g s}=0$ (nonDE gene). We assume that the $Z$-statistics from study $s$ are sampled from a mixture distribution with three mixing components depending on $Y_{g s}: f^{(s)}\left(Z_{g s} \mid Y_{g s}\right)=$ $f_{0}^{(s)}\left(Z_{g s}\right) \cdot \mathbb{I}\left(Y_{g s}=0\right)+f_{+1}^{(s)}\left(Z_{g s}\right) \cdot \mathbb{I}\left(Y_{g s}=1\right)+f_{-1}^{(s)}\left(Z_{g s}\right) \cdot \mathbb{I}\left(Y_{g s}=-1\right)$, where $f^{(s)}(\cdot)$ is the p.d.f. of $Z$-statistics in study $s$, and $f_{0}^{(s)}, f_{+1}^{(s)}$ and $f_{-1}^{(s)}$ are the p.d.f.s of the null, positive and negative components in study $s$.

In most situations, if an appropriate statistical test is adopted, one can expect that $p_{g s} \sim \operatorname{Unif}(0,1)$ if gene $g$ in study $s$ is not DE and hence reasonably assume $f_{0}^{(s)} \equiv \mathrm{N}(0,1)$. If the $p$-value distribution is not uniform under null hypothesis, one can also empirically estimate $f_{0}^{(s)}$ following Efron (2004). Throughout 
this manuscript we use theoretical null $\mathrm{N}(0,1)$, and we put discussion about this choice in the conclusion section. Unlike $f_{0}^{(s)}, f_{ \pm 1}^{(s)}$ are usually unknown, and their estimation is not trivial. To account for the complex composition of alternative $f_{ \pm 1}^{(s)}$ (potentially several subgroups exist in the alternative space), we model them nonparametrically by assuming they are also mixtures of distributions using Dirichlet processes (DPs). DPs are widely discussed and applied in the literature [Neal (2000), Müller and Quintana (2004)], and density estimation using DPs has also been discussed [Escobar and West (1995)]. In our model, when $Y_{g s} \neq 0$, we assume $Z_{g s} \sim \mathrm{N}\left(\mu_{g s}, 1\right)$, and $\mu_{g s}$ follows distribution $G_{s+}$ or $G_{s-\text { generated from }}$ DPs. Specifically, the generative process of $Z_{g s}$ given $Y_{g s}= \pm 1$ is as follows:

$$
\begin{aligned}
G_{s+} & \sim \operatorname{DP}\left(G_{0+}, \alpha_{+}\right) \quad \text { and } \quad G_{s-} \sim \operatorname{DP}\left(G_{0-}, \alpha_{-}\right), \\
\mu_{g s} & \sim \begin{cases}G_{s+} & \text { if } Y_{g_{s}}=1, \\
G_{s-} & \text { if } Y_{g_{s}}=-1,\end{cases} \\
Z_{g s} & \sim \mathrm{N}\left(\mu_{g_{s}}, 1\right) .
\end{aligned}
$$

$\operatorname{DP}(G, \alpha)$ denotes a Dirichlet process with base distribution $G$ and concentration parameter $\alpha$, and $G_{0+}\left(G_{0-}\right)$ denotes normal density $\mathrm{N}\left(0, \sigma_{0}^{2}\right)$ left (right) truncated at 0 . We find that the selection of $\sigma_{0}$ and $\alpha_{ \pm}$do not much affect the performance of this model in simulation (see details in Supplementary Table S3 [Huo, Song and Tseng (2019)]). It should be noted that we assume $Z_{g_{s}} \sim \mathrm{N}\left(\mu_{g_{s}}, 1\right)$, where the variance is fixed at 1 to ensure that $f_{+1}^{(s)} / f_{0}^{(s)}\left(f_{-1}^{(s)} / f_{0}^{(s)}\right)$ is monotonically increasing (decreasing) while $Z_{g s}>0\left(Z_{g s}<0\right)$ which in turn guarantees the posterior probability of gene $g$ being DE in study $s$ to increase as $\left|Z_{g s}\right|$ increases (see Theorem 2.1). In addition this assumption makes the MCMC simpler and hence speeds up the algorithm.

THEOREM 2.1. If $f_{k}(x) \equiv \mathrm{N}\left(\mu_{k}, \sigma_{k}^{2}\right)$ with $\mu_{k}>0, \sigma_{k}^{2} \geq 1$ and $1 \leq k \leq K$, and $f_{0}(x)=\mathrm{N}(0,1)$, then $\sum_{k=1}^{K} w_{k} f_{k} / f_{0}$ is monotonically increasing when $x \geq 0$, where $w_{1}, \ldots, w_{K}>0$ and $\sum_{k=1}^{K} w_{k}=1$.

PROOF. $\forall 1 \leq k \leq K, f_{k}(x)=1 /\left(\sigma_{k} \sqrt{2 \pi}\right) \exp \left(-\left(x-\mu_{k}\right)^{2} /\left(2 \sigma_{k}^{2}\right)\right), f_{0}(x)=$ $1 / \sqrt{2 \pi} \exp \left(-x^{2} / 2\right)$, so $g_{k}(x)=f_{k}(x) / f_{0}(x)=1 / \sigma_{k} \exp \left(-\left(x-\mu_{k}\right)^{2} /\left(2 \sigma_{k}^{2}\right)+\right.$ $\left.x^{2} / 2\right)$ and $\log g_{k}(x)=-\left(x-\mu_{k}\right)^{2} /\left(2 \sigma_{k}^{2}\right)+x^{2} / 2-\log \sigma_{k}$. By taking derivative of $\log g_{k}(x)$, we get $\left[\log g_{k}(x)\right]^{\prime}=-\left(x-\mu_{k}\right) / \sigma_{k}^{2}+x=\left(1-1 / \sigma_{k}^{2}\right) x+\mu_{k} / \sigma_{k}^{2}>0$, when $x \geq 0$ [actually $x>\mu_{k} /\left(1-\sigma_{k}^{2}\right)$ is enough]. Therefore, $g_{k}=f_{k} / f_{0}$ is monotonically increasing when $x \geq 0$, and $\sum_{k=1}^{K} w_{k} f_{k} / f_{0}$ is also monotonically increasing when $x \geq 0$.

In order to borrow information across studies, we further assume that $Y_{g s}$ is generated depending on (1) the prior probability $\pi_{g}$ that gene $g$ is a DE gene 


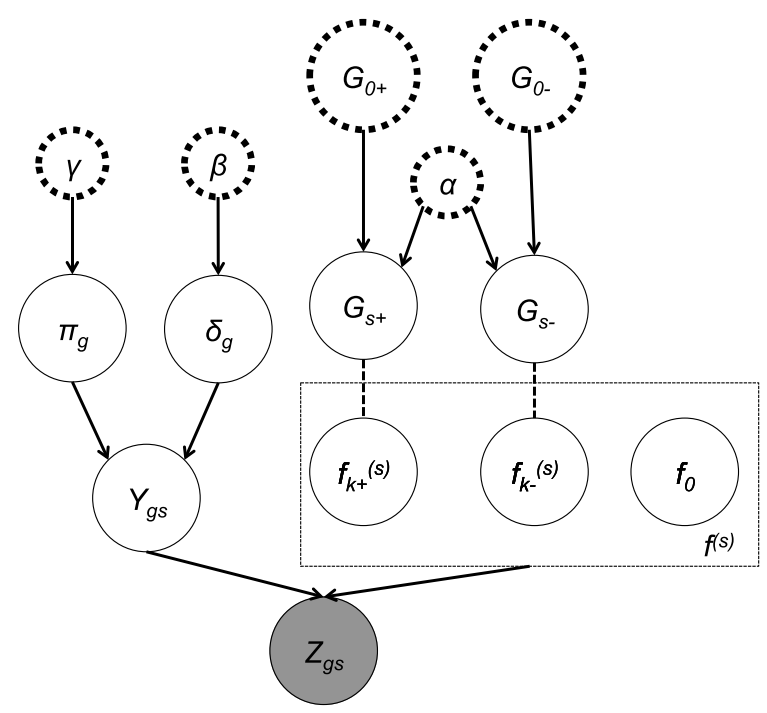

FIG. 2. Graphical representation of Bayesian latent hierarchical model. Shaded nodes are observed variables. Dashed nodes are pre-estimated/fixed parameters. Arrows represent generative process. Dashed lines represent equivalent variables. $s$ is the study index, and $g$ is the gene index.

and (2) the conditional probability $\delta_{g}$ for gene $g$ in study $s$ being up-regulated (or $1-\delta_{g}$ for down-regulated), given gene $g$ is DE. Specifically, we assume $\vec{W}_{g s} \sim \operatorname{Mult}\left(1,\left(1-\pi_{g}, \pi_{g}^{+}, \pi_{g}^{-}\right)\right)$and $Y_{g s}=\vec{W}_{g s} \cdot(0,1,-1)$, where $\pi_{g}^{+}=\pi_{g} \delta_{g}$, $\pi_{g}^{-}=\pi_{g}\left(1-\delta_{g}\right)$ and $\cdot$ is the inner product of two vectors. Given $Y_{g s}=y, Z_{g s}$ is generated from $f_{y}^{(s)}(Z)$. The graphical representation of the full generative model is shown in Figure 2.

We assume that each gene $g$ is DE in different studies in the same probability $\pi_{g}$, that is, $\pi_{g}=\operatorname{Pr}\left(Y_{g s} \neq 0\right)$, and $\pi_{g} \sim \operatorname{Beta}(\gamma, 1-\gamma) \cdot \gamma$ can be interpreted as the proportion of DE genes pooling all studies, since the expectation of $\pi_{g}$ from this prior is $\gamma$. We further set the prior of $\gamma$ being uniform distribution $[\gamma \sim \operatorname{UNIF}(0,1)]$.

For each gene $g$, define $\delta_{g}=\operatorname{Pr}\left(Y_{g s}=1 \mid\right.$ gene $g$ is a DE gene $)$. We assume $\delta_{g} \sim \operatorname{Beta}(\beta, \beta)$. We set $\beta=1 / 2$ in this paper which gives a noninformative prior. Note that this conditional probability provides flexibility for a DE gene to contain conflicting differential expression directions (i.e., up-regulation in one study but down-regulation in another study; e.g., module III in Figure 1).

2.2. Model fitting. Since conjugate priors were used in the generative model, we can generate the posterior samples efficiently using the MCMC procedure. In order to update the DP with an infinite number of components, we take the alternative view of DP as the Chinese restaurant process. We define $C_{g s} \in$ $\{\ldots,-3,-2,-1,0,1,2,3, \ldots\}$ as the auxiliary component variable, and $Y_{g s}$ is 
determined by the sign of $C_{g s}$. Specifically, if $C_{g s}=0$, we set $Y_{g s}=0$; if $C_{g_{s}}=k$ with $k>0$, then we set $Y_{g s}=1$, and sample $\mu_{g s}$ from the $k$ th component (or $k$ th table in Chinese restaurant process) of $G_{s+}$, and similarly, if $C_{g_{s}}=k$ with $k<0$, then we set $Y_{g s}=-1$ and sample $\mu_{g s}$ from the $k$ th component of $G_{s-}$. The following steps provide details of our MCMC iterations:

1. Update $\pi_{g}$ 's:

$$
\pi_{g} \mid Y_{g s} \sim \operatorname{Beta}\left(\gamma+Y_{g}^{+}+Y_{g}^{-}, S-Y_{g}^{+}-Y_{g}^{-}+1-\gamma\right),
$$

where $Y_{g}^{+}=\sum_{s} \mathbb{I}\left(Y_{g s}=1\right)$ and $Y_{g}^{-}=\sum_{s} \mathbb{I}\left(Y_{g s}=-1\right)$.

2. Update $\delta_{g}$ 's:

$$
\delta_{g} \mid Y_{g s} \sim \operatorname{Beta}\left(\beta+Y_{g}^{+}, \beta+Y_{g}^{-}\right) .
$$

3. Update $Y_{g s}$ 's:

First update $C_{g s}$ 's s.t.

$$
\operatorname{Pr}\left(C_{g s}=k \mid C_{-g, s}, Z_{g s}, \pi_{g}^{ \pm}\right) \propto h_{k}^{(s)}\left(Z_{g s} \mid C_{-g, s}\right)\left(\pi_{g}^{+}\right)^{\mathbb{I}(k>0)}\left(\pi_{g}^{-}\right)^{\mathbb{I}(k<0)}\left(1-\pi_{g}\right)^{\mathbb{I}(k=0)},
$$

where $C_{-g, s}$ denotes all the $C$ 's in study $s$ excluding gene $g$. Note that $h_{k}^{(s)}$ can be calculated directly following the convention of algorithm 3 in Neal (2000). Details of $h_{k}^{(s)}\left(Z_{g s} \mid C_{-g, s}\right)$ are given in Supplementary Section III [Huo, Song and Tseng (2019)]. Finally, we set $Y_{g s}=\operatorname{sgn}\left(C_{g s}\right)$, where $\operatorname{sgn}(\cdot)$ is the sign function.

4. Because there is no immediate conjugate prior for $\gamma$, we sample $\gamma$ using the Metropolis-Hastings algorithm such that

$$
\gamma \propto \prod_{g=1}^{G} \operatorname{dBeta}\left(\pi_{g} ; \gamma, 1-\gamma\right),
$$

where $\operatorname{dBeta}(x ; a, b)$ is the probability density function of beta distribution evaluated at $x$ with the shape parameters $a$ and $b$. For details see Supplementary Section I [Huo, Song and Tseng (2019)].

2.3. Decision space and inference making. A main benefit of Bayesian modeling is its capability of making inference by statistical decision theory, a generalized framework that covers the traditional hypothesis testing framework as a special case [Berger (2013)]. Take $\mathrm{HS}_{\bar{A}}$ from Section 1 as an example. Traditional hypothesis testing considers null hypothesis $H_{0}: \vec{\theta}_{g} \in \Omega_{\bar{A}}^{0}$, where $\Omega_{\bar{A}}^{0}=$ $\left\{\vec{\theta}_{g}: \sum_{s=1}^{S} \mathbb{I}\left(\theta_{g s} \neq 0\right)<S\right\}$ and $\vec{\theta}_{g}=\left(\theta_{g 1}, \ldots, \theta_{g S}\right)$, versus alternative hypothesis $H_{A}: \vec{\theta}_{g} \in \Omega_{\bar{A}}^{1}$ where $\Omega_{\bar{A}}^{1}=\left\{\vec{\theta}_{g}: \sum_{S=1}^{S} \mathbb{I}\left(\theta_{g s} \neq 0\right)=S\right\}$. When observed data are unlikely to happen (i.e., type I error controlled at $5 \%$ ) under null hypothesis, we reject the null hypothesis. One notable feature is that traditional hypothesis testing views null and alternative hypothesis spaces differently. The decision of 
hypothesis testing is only based on the null hypothesis-either to reject or to accept. The alternative hypothesis plays little role in decision making. In view of decision theory framework, a decision space (aka action space) is designed as $\mathcal{D}_{\bar{A}}=\left(\Omega_{\bar{A}}^{0}, \Omega_{\bar{A}}^{1}\right)$. The inference generates a decision function $f$ that maps from the observed data space $Z$ to $\mathcal{D}_{\bar{A}}$ (i.e., $f: Z \rightarrow \mathcal{D}_{\bar{A}}$ ). Under this framework the type I error can be expressed as $\operatorname{Pr}\left(f(Z)=\Omega_{\bar{A}}^{1} \mid \vec{\theta}_{g} \in \Omega_{\bar{A}}^{0}\right)$, and the type II error as $\operatorname{Pr}\left(f(Z)=\Omega_{\bar{A}}^{0} \mid \vec{\theta}_{g} \in \Omega_{\bar{A}}^{1}\right)$. Hypothesis testing is a special case under this framework with adequate type I error control to determine the decision function $f$. Unlike hypothesis testing, decision theory treats $\Omega_{\bar{A}}^{0}$ and $\Omega_{\bar{A}}^{1}$ equally, because in decision theory the decision is made through cost analysis which weighs the costs of making wrong decisions in both spaces. One can easily design a realistic loss (cost) function based on the two types of errors to determine their balance and achieve the best decision function. In this paper in order to make a fair comparison with classical hypothesis testing, we use posterior probabilities from Bayesian modeling and adopt a false discovery control described by Newton et al. (2004) to determine the decision function.

Denote by $\xi_{g}=\operatorname{Pr}\left(\vec{\theta}_{g} \in \Omega_{\bar{A}}^{0} \mid Z\right)=1-\operatorname{Pr}\left(\vec{\theta}_{g} \in \Omega_{\bar{A}}^{1} \mid Z\right)$ which is local FDR [Efron and Tibshirani (2002)] by definition. Given a threshold $\kappa$, we declare gene $g$ as a DE gene if $\xi_{g} \leq \kappa$, and the expected number of false discoveries is $\sum_{g} \xi_{g} \mathbb{I}\left(\xi_{g} \leq \kappa\right)$. The false discovery rate from Bayesian modeling is defined as $\frac{\sum_{g} \xi_{g} \mathbb{I}\left(\xi_{g} \leq \kappa\right)}{\sum_{g} \mathbb{I}\left(\xi_{g} \leq \kappa\right)}$ [Newton et al. (2004)]. In simulation and real data applications, we compare the performance of FDR control from traditional hypothesis testing and FDR control from Bayesian modeling. Note that we can consider $\mathcal{D}_{B}=\left(\Omega_{B}^{0}, \Omega_{B}^{1}\right)$, where $\Omega_{B}^{0}=\left\{\vec{\theta}_{g}: \sum_{s=1}^{S} \mathbb{I}\left(\theta_{g s} \neq 0\right)=0\right\}$ and $\Omega_{B}^{1}=\left\{\vec{\theta}_{g}: \sum_{s=1}^{S} \mathbb{I}\left(\theta_{g s} \neq 0\right)>0\right\}$, to correspond to $\mathrm{HS}_{B}$; and $\mathcal{D}_{\bar{r}}=\left(\Omega_{\bar{r}}^{0}, \Omega_{\bar{r}}^{1}\right)$, where $\Omega_{\bar{r}}^{0}=\left\{\vec{\theta}_{g}: \sum_{S=1}^{S} \mathbb{I}\left(\theta_{g s} \neq 0\right)<r\right\}$ and $\Omega_{\bar{r}}=\left\{\vec{\theta}_{g}: \sum_{s=1}^{S} \mathbb{I}\left(\theta_{g s} \neq 0\right) \geq r\right\}$, to correspond to $\mathrm{HS}_{\bar{r}}$.

Finally, for a declared DE gene we are given the posterior probability of whether gene $g$ in study $s$ is a non-DE gene $\left[\operatorname{Pr}\left(Y_{g s}=0 \mid Z\right)\right]$, an up-regulated gene $\left[\operatorname{Pr}\left(Y_{g s}=1 \mid Z\right)\right]$ or a down-regulated gene $\left[\operatorname{Pr}\left(Y_{g s}=-1 \mid Z\right)\right]$. We propose a gene- and study-specific confidence score $V_{g s}=\operatorname{Pr}\left(Y_{g_{s}}=1 \mid Z\right)-\operatorname{Pr}\left(Y_{g s}=-1 \mid Z\right)$ which ranges between -1 and 1 . We are confident that gene $g$ is up-regulated in study $s$, if $V_{g s}$ is close to 1 , and vice versa when $V_{g s}$ is close to -1 . See Figure 1(b) for an example.

2.4. Biomarker clustering for meta-patterns of homogenous and heterogenous differential signals. Several recently developed meta-analysis methods [Li and Tseng (2011), Bhattacharjee et al. (2012), Li et al. (2014)] provide modeling of homogeneous and heterogeneous differential signals. The 0 or 1 differential expression indicators (e.g., $\vec{w}_{g}$ in AW-Fisher) allow further biological investigation on consensus biomarkers as well as study-specific biomarkers. However, when $S$ becomes large, the number of biomarker categories grows exponentially to $2^{S}-1$, 
and the biomarker categories become intractable. In BayesMP the posterior probability of the differential expression indicator [i.e., $\left.\operatorname{Pr}\left(Y_{g s} \mid Z\right)\right]$ provides probabilistic soft conclusions. After we obtain a list of biomarkers under certain global FDR control (e.g., $5 \%$ or $1 \%$ ), we apply the tight clustering algorithm [Tseng and Wong (2005)] to generate data-driven biomarker modules. Tight clustering is a resampling-based algorithm built upon $K$-means or $K$-medoids. This method aggregates information from repeated clustering of subsampled data to directly identify tight clusters (i.e., sets of biomarkers with small dissimilarity) and does not force every biomarker into a cluster. It can be applied to any dissimilarity matrix if $K$-medoids is used. Pathway enrichment analysis is then applied to functionally annotate each biomarker module. The resulting biomarker modules of different meta-patterns will greatly facilitate interpretation and hypothesis generation for further biological investigation. In the first two real data applications, for example, heterogeneous meta-patterns of biomarkers are expected from the nature of multitissue or multibrain-region design across studies. Biomarkers up-regulated in one brain region but non-DE (or even down-regulated) in another brain region is of great interest. It should be noted that in the third breast cancer data application meta-patterns still help characterize the heterogeneity of different cohorts (e.g., differences of study population and probe design), even though this heterogeneity is hopefully minimal since these studies focus on the same disease with homogeneous tissue type.

To apply tight clustering, we need to define a dissimilarity measure for any pair of genes. Denote by $\vec{U}_{g s}$ the posterior probability vector for $Y_{g s}: \vec{U}_{g s}=$ $\left(\operatorname{Pr}\left(Y_{g s}=1 \mid Z\right), \operatorname{Pr}\left(Y_{g s}=-1 \mid Z\right), \operatorname{Pr}\left(Y_{g s}=0 \mid Z\right)\right)$ which can be estimated by MCMC samples. For two genes $i$ and $j$, we first calculate the dissimilarity of $\vec{U}_{i s}$ and $\vec{U}_{j s}$ in study $s$ and then average over study index $s$. The dissimilarity measure between $\vec{U}_{i s}$ and $\vec{U}_{j s}$ we considered includes cosine dissimilarity, $l_{2}$ dissimilarity, $l 2_{2 D}$ dissimilarity, symmetric KL dissimilarity and Hellinger dissimilarity. Definitions and details of these dissimilarity measurements are in Supplementary Section II [Huo, Song and Tseng (2019)]. By using the simulation setting in Section 3.2, we found cosine dissimilarity outperforms others (see details in Supplementary Figure S3 [Huo, Song and Tseng (2019)]), and hence adopted it in our paper and would recommend it for other applications.

\section{Simulation results.}

3.1. DE gene detection and FDR control. To evaluate the performance of the proposed method and compare to other methods, we performed the simulations below:

1. Let $S$ be the number of studies, $G=10,000$ be the total number of genes, and $N=20$ be the number of cases and controls ( $2 N$ samples in total). 
2. We firstly focus on simulating gene correlation structure and assume no effect size for all genes in all studies. We sample expression levels with correlated genes following the procedure in Song and Tseng (2014):

(a) Sample 200 gene clusters with 20 genes in each cluster, and the remaining 6000 genes are uncorrelated. Denote by $C_{g} \in\{0,1, \ldots, 200\}$ the cluster membership indicator for gene $g$, for example, $C_{g}=1$ indicates gene $g$ is in cluster 1 , whereas $C_{g}=0$ indicates gene $g$ is not in any gene cluster.

(b) For cluster $c$ and study $s$, sample $A_{c s}^{\prime} \sim \mathrm{W}^{-1}(\Psi, 60)$, where $1 \leq c \leq 200$, $\Psi=0.5 I_{20 \times 20}+0.5 J_{20 \times 20}, \mathrm{~W}^{-1}$ denotes the inverse Wishart distribution, $I$ is the identity matrix and $J$ is the matrix with all elements equal to 1 . Then $A_{c s}$ is calculated by standardizing $A_{c s}^{\prime}$ such that the diagonal elements are all $1 \mathrm{~s}$. The covariance matrix for gene cluster $c$ in study $s$ is calculated as $\Sigma_{c s}=\sigma^{2} A_{c s}$, where $\sigma$ is a tuning parameter we vary in the evaluation.

(c) Denote by $g_{c 1}, \ldots, g_{c 20}$ the indices of the 20 genes in cluster $c$ [i.e., $C_{g_{c j}}=c$, where $1 \leq c \leq C(C=200)$, and $\left.1 \leq j \leq 20\right]$. Sample expression levels of genes in cluster $c$ for sample $n$ in study $s$ as $\left(X_{g_{c 1} s n}^{\prime}, \ldots, X_{g_{c 20} s n}^{\prime}\right) \sim$ $\operatorname{MVN}\left(0, \Sigma_{c s}\right)$, where $1 \leq n \leq 2 N$ and $1 \leq s \leq S$. For any uncorrelated gene $g$ with $C_{g}=0$, sample the expression level for sample $n$ in study $s$ as $X_{g s n}^{\prime} \sim \mathrm{N}\left(0, \sigma^{2}\right)$, where $1 \leq n \leq 2 N$ and $1 \leq s \leq S$.

3. Sample DE genes, effect sizes and their differential expression directions:

(a) Assume that the first $G_{1}$ genes are DE in at least one of the combined studies, where $G_{1}=30 \% \times G$. For each $1 \leq g \leq G_{1}$, sample $v_{g}$ from discrete uniform distribution $v_{g} \sim \operatorname{UNIF}(1, \ldots, S)$ and then randomly sample subset $\mathbf{v}_{g} \subseteq\{1, \ldots, S\}$ such that $\left|\mathbf{v}_{g}\right|=v_{g}$. Here $\mathbf{v}_{g}$ is the set of studies in which gene $g$ is DE.

(b) For any DE gene $g\left(1 \leq g \leq G_{1}\right)$, sample gene-level effect size $\theta_{g} \sim$ $\mathrm{N}_{0.5+}(1,1)$, where $\mathrm{N}_{a+}$ denotes the truncated Gaussian distribution within interval $(a, \infty)$. For any $s \in \mathbf{v}_{g}$, also sample study-specific effect size $\theta_{g s} \sim \mathrm{N}_{0+}\left(\theta_{g}, 0.2^{2}\right)$.

(c) Sample $d_{g} \sim \operatorname{Ber}(0.5)$, where $1 \leq g \leq G_{1}$ and $s \in \mathbf{v}_{g}$. Here $d_{g}$ controls effect size direction for gene $g$.

4. Add the effect sizes to the gene expression levels sampled in step (c). For controls $(1 \leq n \leq N)$, set the expression levels as $X_{g s n}=X_{g s n}^{\prime}$. For cases $(N+1 \leq$ $n \leq 2 N)$, if $1 \leq g \leq G_{1}$ and $s \in \mathbf{v}_{g}$, set the expression levels as $X_{g s n}=X_{g s n}^{\prime}+$ $(-1)^{d_{g}} \theta_{g s}$; otherwise, set $X_{g s n}=X_{g s n}^{\prime}$.

We performed simulation with $S=3,5,10$ and $\sigma=1,2,3$ to account for different numbers of combined studies and various signal/noise ratio. We applied limma to compare the gene expression levels between the control group and the case group. We transformed the two-sided $p$-values from limma to one-sided $p$-values by taking account of the directions of estimated effect sizes. Then one-sided $p$-values are transformed to $Z$ statistics. BayesMP took 53 minutes on a regular PC with 
1.4 GHz CPU (i.e., for one simulation with $S=3$ and $\sigma=1$ ) to obtain 10,000 posterior samples using MCMC. Supplementary Figure S1 [Huo, Song and Tseng (2019)] shows the posterior samples of $\pi_{g}$ in two example genes-a DE gene and a non-DE gene as well as $\gamma$. Because the posterior samples converge to a stationary distribution very quickly for our method (see examples in Supplementary Figure S1 [Huo, Song and Tseng (2019)]), excluding 500 posterior samples for burn-in is enough for the analyses of this paper. We repeated the simulation 100 times and averaged the results. We compared the performance of our method and existing methods designed for decision space $\mathcal{D}_{\bar{A}}$ (maxP), $\mathcal{D}_{B}$ (Fisher's method and $\mathrm{AW}$ ) and $\mathcal{D}_{\bar{r}}$ (rOP) with $r=\lfloor S / 2\rfloor+1$, using false discovery rate (FDR), false negative rate (FNR) and the area under the curve (AUC) of the receiver operating characteristic (ROC) curve. Note that in our comparison we used $\mathcal{D}_{\bar{A}}$ and $\mathcal{D}_{\bar{r}}$, which are equivalent to the complementary hypothesis testings $\mathrm{HS}_{\bar{A}}$ and $\mathrm{HS}_{\bar{r}}$, and the true number of studies in which a gene is DE can be calculated because the truth is known in simulation. Table 1 compares the FDR, FNR and AUC of different methods at nominal FDR level 5\% which is widely accepted in genomic research. For decision space $\mathcal{D}_{B}$ all the three methods controlled FDR around its nominal level which is anticipated because $\mathrm{HS}_{B}$ is complementary and equivalent to $\mathcal{D}_{B}$. Fisher and AW were slightly overconservative in terms of FDR control; Fisher's method and AW controlled FDR at around 3.5\%, whereas our BayesMP controlled FDR at around the nominal 5\%. This phenomenon has also been observed in Song and Tseng (2014) when the genes are correlated. Because BayesMP is less conservative than the other two methods, we were able to detect slightly more genes under $\mathcal{D}_{B}$. In addition BayesMP achieved similar (or slightly better) FNR and AUC with Fisher and AW under $\mathcal{D}_{B}$. Fisher' s method is known to be almost optimal, that is, Fisher' s method achieves asymptotic Bahadur optimality (ABO) [Littell and Folks (1971)] when effect sizes are consistent and equal for all studies. This indicated BayesMP is also almost optimal for $\mathcal{D}_{B}$ under the simulation scenario. For decision space $\mathcal{D}_{\bar{A}}$ and $\mathcal{D}_{\bar{r}}$ we observed that maxP and rOP lost control of FDR. As discussed in Section 1, this is caused by the nature that $\mathrm{HS}_{A}$ and $\mathrm{HS}_{r}$ have noncomplementary null and alternative spaces. To the contrary, BayesMP still controlled FDR close to its nominal level for $\mathcal{D}_{\bar{A}}$ and $\mathcal{D}_{\bar{r}}$. Note that because maxP and rOP were not able to control FDR at its nominal level, the number of genes detected by these methods was not directly comparable to our methods. However, FNR of BayesMP was only slightly larger than rOP for $\mathcal{D}_{\bar{r}}$ regardless of the conservative FDR control. When $S$ was large $(S=10)$ in simulation, the FDR control of BayesMP under $\mathcal{D}_{\bar{A}}$ deviated from its nominal level (around 10\% instead of 5\%). The reason for the anticonservative control was that the data simulation setting was different from model generative process, thus small errors accumulated when $S$ got large. However, BayesMP still performed much better than maxP and $\operatorname{roP}\left(\mathrm{FDR}=0.58\right.$ for $\operatorname{maxP}$ in $\mathcal{D}_{\bar{A}}$ and $\mathrm{FDR}=0.23$ for rOP in $\mathcal{D}_{\bar{r}}$ setting). In addition BayesMP achieved much larger AUC than maxP and rOP under $\mathcal{D}_{\bar{A}}$ and $\mathcal{D}_{\bar{r}}$ which indicated better predictive power of BayesMP. 
TABLE 1

Comparison of different methods by FDR, FNR and AUC of ROC curve for decision spaces $\mathcal{D}_{\bar{A}}$, $\mathcal{D}_{B}$ and $\mathcal{D}_{\bar{r}}$. The nominal FDR is $5 \%$ for all compared methods. The mean results and $S D$ (in parentheses) were calculated based on 100 simulations

\begin{tabular}{|c|c|c|c|c|c|c|c|c|c|}
\hline & \multirow[b]{2}{*}{$S$} & \multirow[b]{2}{*}{$\sigma$} & \multicolumn{2}{|c|}{$\mathcal{D}_{\bar{A}}$} & \multicolumn{3}{|c|}{$\mathcal{D}_{B}$} & \multicolumn{2}{|c|}{$\mathcal{D}_{\bar{r}}(r=\lfloor S / 2\rfloor+1)$} \\
\hline & & & BayesMP & $\max P$ & BayesMP & Fisher & AW & BayesMP & rOP \\
\hline \multirow{16}{*}{ FDR } & \multirow{4}{*}{3} & 1 & $\begin{array}{c}0.058 \\
(0.008)\end{array}$ & $\begin{array}{c}0.207 \\
(0.014)\end{array}$ & $\begin{array}{c}0.042 \\
(0.004)\end{array}$ & $\begin{array}{c}0.035 \\
(0.005)\end{array}$ & $\begin{array}{c}0.035 \\
(0.004)\end{array}$ & $\begin{array}{c}0.034 \\
(0.004)\end{array}$ & $\begin{array}{c}0.086 \\
(0.007)\end{array}$ \\
\hline & & 2 & $\begin{array}{c}0.058 \\
(0.010)\end{array}$ & $\begin{array}{c}0.198 \\
(0.017)\end{array}$ & $\begin{array}{c}0.047 \\
(0.006)\end{array}$ & $\begin{array}{c}0.035 \\
(0.006)\end{array}$ & $\begin{array}{c}0.036 \\
(0.006)\end{array}$ & $\begin{array}{c}0.037 \\
(0.005)\end{array}$ & $\begin{array}{r}0.080 \\
(0.009)\end{array}$ \\
\hline & & & 0.043 & 0.184 & 0.050 & 0.035 & 0.036 & 0.036 & 0.073 \\
\hline & & 3 & $(0.016)$ & $(0.025)$ & $(0.009)$ & $(0.008)$ & $(0.009)$ & $(0.009)$ & $(0.014)$ \\
\hline & \multirow{6}{*}{5} & 1 & 0.075 & 0.361 & 0.043 & 0.034 & 0.034 & 0.037 & 0.130 \\
\hline & & & $(0.011)$ & $(0.017)$ & $(0.004)$ & $(0.004)$ & (0.004) & $(0.005)$ & $(0.008)$ \\
\hline & & \multirow{2}{*}{2} & 0.079 & 0.349 & 0.046 & 0.034 & 0.034 & 0.042 & 0.115 \\
\hline & & & (0.019) & $(0.022)$ & $(0.006)$ & $(0.005)$ & $(0.005)$ & $(0.006)$ & $(0.010)$ \\
\hline & & 3 & 0.062 & 0.330 & 0.050 & 0.034 & 0.034 & 0.042 & 0.099 \\
\hline & & & $(0.033)$ & $(0.028)$ & $(0.007)$ & $(0.006)$ & $(0.007)$ & $(0.008)$ & $(0.013)$ \\
\hline & \multirow{6}{*}{10} & 1 & 0.105 & 0.580 & 0.050 & 0.035 & 0.035 & 0.047 & 0.231 \\
\hline & & & $(0.017)$ & $(0.021)$ & $(0.003)$ & $(0.004)$ & $(0.004)$ & $(0.005)$ & $(0.010)$ \\
\hline & & 2 & 0.122 & 0.569 & 0.050 & 0.035 & 0.035 & 0.059 & 0.200 \\
\hline & & & $(0.029)$ & $(0.027)$ & $(0.005)$ & $(0.005)$ & $(0.005)$ & $(0.007)$ & $(0.012)$ \\
\hline & & 3 & 0.108 & 0.554 & 0.054 & 0.035 & 0.035 & 0.062 & 0.167 \\
\hline & & & $(0.064)$ & (0.044) & $(0.007)$ & $(0.006)$ & $(0.006)$ & (0.010) & $(0.015)$ \\
\hline \multirow{18}{*}{ FNR } & \multirow{6}{*}{3} & 1 & 0.024 & 0.017 & 0.054 & 0.058 & 0.056 & 0.039 & 0.032 \\
\hline & & 1 & $(0.002)$ & $(0.001)$ & $(0.003)$ & $(0.003)$ & $(0.003)$ & $(0.002)$ & $(0.002)$ \\
\hline & & \multirow{2}{*}{2} & 0.064 & 0.055 & 0.170 & 0.181 & 0.183 & 0.114 & 0.112 \\
\hline & & & $(0.002)$ & $(0.002)$ & $(0.003)$ & $(0.003)$ & $(0.003)$ & $(0.003)$ & $(0.003)$ \\
\hline & & \multirow{2}{*}{3} & 0.089 & 0.082 & 0.240 & 0.253 & 0.257 & 0.161 & 0.166 \\
\hline & & & $(0.003)$ & $(0.002)$ & $(0.002)$ & $(0.002)$ & $(0.002)$ & $(0.003)$ & $(0.003)$ \\
\hline & \multirow{6}{*}{5} & \multirow[t]{2}{*}{1} & 0.016 & 0.009 & 0.043 & 0.048 & 0.045 & 0.032 & 0.021 \\
\hline & & & $(0.001)$ & $(0.001)$ & $(0.003)$ & $(0.002)$ & $(0.003)$ & $(0.002)$ & $(0.001)$ \\
\hline & & 2 & 0.040 & 0.031 & 0.150 & 0.162 & 0.164 & 0.092 & 0.086 \\
\hline & & 2 & $(0.002)$ & $(0.002)$ & (0.003) & $(0.003)$ & $(0.003)$ & $(0.002)$ & $(0.002)$ \\
\hline & & 3 & 0.054 & 0.047 & 0.219 & 0.236 & 0.241 & 0.132 & 0.136 \\
\hline & & 5 & $(0.002)$ & $(0.002)$ & $(0.003)$ & $(0.003)$ & $(0.003)$ & $(0.002)$ & $(0.003)$ \\
\hline & & 1 & 0.009 & 0.004 & 0.030 & 0.035 & 0.031 & 0.027 & 0.010 \\
\hline & & & $(0.001)$ & $(0.001)$ & $(0.002)$ & $(0.002)$ & $(0.002)$ & $(0.001)$ & $(0.001)$ \\
\hline & 10 & 2 & 0.022 & 0.015 & 0.119 & 0.132 & 0.132 & 0.070 & 0.054 \\
\hline & 10 & 2 & (0.001) & $(0.001)$ & $(0.003)$ & $(0.003)$ & $(0.003)$ & $(0.002)$ & $(0.002)$ \\
\hline & & 3 & 0.028 & 0.023 & 0.184 & 0.206 & 0.211 & 0.097 & 0.095 \\
\hline & & & $(0.002)$ & $(0.002)$ & (0.003) & $(0.002)$ & $(0.003)$ & (0.003) & $(0.003)$ \\
\hline
\end{tabular}


TABLE 1

(Continued)

\begin{tabular}{|c|c|c|c|c|c|c|c|c|c|}
\hline & \multirow[b]{2}{*}{$S$} & \multirow[b]{2}{*}{$\sigma$} & \multicolumn{2}{|c|}{$\mathcal{D}_{\bar{A}}$} & \multicolumn{3}{|c|}{$\mathcal{D}_{B}$} & \multicolumn{2}{|c|}{$\mathcal{D}_{\bar{r}}(r=\lfloor S / 2\rfloor+1)$} \\
\hline & & & BayesMP & $\max P$ & BayesMP & Fisher & AW & BayesMP & rOP \\
\hline \multirow{16}{*}{ AUC } & \multirow{5}{*}{3} & 1 & $\begin{array}{c}0.976 \\
(0.003)\end{array}$ & $\begin{array}{c}0.926 \\
(0.003)\end{array}$ & $\begin{array}{c}0.973 \\
(0.002)\end{array}$ & $\begin{array}{c}0.973 \\
(0002)\end{array}$ & $\begin{array}{c}0.973 \\
(0.002)\end{array}$ & $\begin{array}{c}0.980 \\
(0.002)\end{array}$ & $\begin{array}{r}0.972 \\
(0.003)\end{array}$ \\
\hline & & $?$ & 0.906 & 0.876 & 0.880 & 0.878 & 0.876 & 0.902 & 0.873 \\
\hline & & 2 & $(0.006)$ & $(0.006)$ & $(0.005)$ & $(0.005)$ & $(0.005)$ & $(0.005)$ & $(0.006)$ \\
\hline & & 3 & 0.833 & 0.806 & 0.788 & 0.784 & 0.780 & 0.820 & 0.776 \\
\hline & & & (0.008) & $(0.008)$ & (0.006) & $(0.006)$ & $(0.006)$ & (0.007) & $(0.008)$ \\
\hline & \multirow{5}{*}{5} & 1 & $\begin{array}{c}0.974 \\
(0.004)\end{array}$ & $\begin{array}{c}0.920 \\
(0.003)\end{array}$ & $\begin{array}{c}0.978 \\
(0.002)\end{array}$ & $\begin{array}{c}0.978 \\
(0.002)\end{array}$ & $\begin{array}{c}0.979 \\
(0.002)\end{array}$ & $\begin{array}{c}0.985 \\
(0.002)\end{array}$ & $\begin{array}{r}0.979 \\
(0.002)\end{array}$ \\
\hline & & $?$ & 0.918 & 0.891 & 0.896 & 0.893 & 0.892 & 0.928 & 0.893 \\
\hline & & 2 & $(0.007)$ & $(0.006)$ & $(0.004)$ & $(0.004)$ & $(0.004)$ & $(0.004)$ & $(0.004)$ \\
\hline & & 3 & 0.866 & 0.833 & 0.812 & 0.805 & 0.800 & 0.859 & 0.801 \\
\hline & & 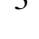 & (0.009) & $(0.009)$ & $(0.005)$ & $(0.005)$ & $(0.006)$ & $(0.005)$ & $(0.006)$ \\
\hline & \multirow{6}{*}{10} & 1 & 0.964 & 0.910 & 0.985 & 0.983 & 0.985 & 0.985 & 0.986 \\
\hline & & & (0.007) & $(0.003)$ & $(0.002)$ & $(0.002)$ & $(0.002)$ & $(0.002)$ & $(0.002)$ \\
\hline & & 2 & 0.907 & 0.905 & 0.920 & 0.917 & 0.917 & 0.948 & 0.920 \\
\hline & & 2 & $(0.010)$ & $(0.006)$ & $(0.004)$ & (0.004) & $(0.004)$ & (0.004) & $(0.004)$ \\
\hline & & 3 & 0.883 & 0.865 & 0.849 & 0.840 & 0.835 & 0.907 & 0.838 \\
\hline & & $J$ & (0.011) & $(0.009)$ & $(0.005)$ & $(0.005)$ & (0.005) & $(0.005)$ & $(0.006)$ \\
\hline
\end{tabular}

3.2. Simulation to evaluate meta-pattern gene module detection. To evaluate the performance of gene module detection, we adopted a simulation procedure similar to Section 3.1. We simulated $S=4$ studies in total. Among the $G=10,000$ genes, we set $4 \%$ of them as homogeneously concordant DE genes with the same direction in all studies (all positive or all negative). We denote "homo+" as the homogeneously concordant DE genes with all positive effect sizes and "homo-" as the homogeneously concordant DE genes with all negative effect sizes. We also set another $4 \%$ of all genes as study-specific DE genes-differentially expressed only in one study. Among them $1 / 4$ are DE genes only in the first study with positive effect sizes (denoted as "ssp1+"), $1 / 4$ are DE genes only in the first study with negative effect sizes (denoted as "ssp1-"), 1/4 are DE genes only in the second study with positive effect sizes (denoted as "ssp2+") and the remaining $1 / 4$ are DE genes only in the second study with negative effect sizes (denoted as "ssp2-"). The rest of the genes are not DE (denoted as "non-DE"). The biological variance $\sigma$ is set to 1 in this simulation.

We first applied the proposed method to this synthetic dataset. We controlled FDR at 5\% under $\mathcal{D}_{B}$ and obtained 691 genes. These genes were used as input for our gene module detection using the tight clustering algorithm. We identified six gene modules in these 691 genes. The detected gene modules are tabulated 
TABLE 2

Contingency table of simulation underlying truth and tight clustering result with six target modules. Zero represents the scattered gene group. 1 6 represent six detected modules.

Bolded numbers are genes with correct assignment

\begin{tabular}{lrrrrrrr}
\hline Module & homo- & homo+ & ssp1- & ssp1+ & ssp2- & ssp2+ & non-DE \\
\hline 1 & $\mathbf{1 7 7}$ & 0 & 0 & 0 & 0 & 0 & 0 \\
2 & 0 & $\mathbf{1 6 4}$ & 0 & 0 & 0 & 0 & 0 \\
3 & 0 & 2 & 0 & 0 & 0 & $\mathbf{8 4}$ & 4 \\
4 & 0 & 4 & 0 & $\mathbf{7 2}$ & 0 & 0 & 6 \\
5 & 0 & 0 & $\mathbf{6 6}$ & 0 & 0 & 0 & 2 \\
6 & 1 & 0 & 0 & 0 & $\mathbf{6 2}$ & 0 & 0 \\
0 & 6 & 4 & 0 & 0 & 7 & 0 & $\mathbf{1 9}$ \\
\hline
\end{tabular}

against the true gene modules simulated in Table 2 (module 0 contains scattered genes not assigned to any of the six modules). The detected gene modules clearly correspond to the true modules, and most of the non-DE genes were left to the noises. The heatmaps, confidence scores and DE patterns of these six modules are shown in Supplementary Figure S2 [Huo, Song and Tseng (2019)]. An alternative approach is to apply tight clustering directly on the $Z$-statistics. By comparing the results, we found that the modules detected by this naive approach are neither pure nor distinguishable under our simulation settings (see details in Supplementary Table S1 [Huo, Song and Tseng (2019)]).

3.3. Additional simulations on sample size effects. To assess impact of unbalanced sample size, we simulated the following special scenarios with:

1. different numbers of samples in different studies,

2. different numbers of cases and controls in each study,

3. different ratios of case and control samples in each study.

Below, we followed the simulation setting in Section 3.1 unless otherwise mentioned. In scenario 1 we allowed different studies to have different numbers of samples. Under this scenario we simulated case (a) with the numbers of samples (case/control) being 20/20, 30/30, 40/40 for three studies respectively, and case (b) with the numbers of samples (case/control) being 20/20, 50/50, 100/100 respectively. In scenario 2 we allowed the numbers of cases and controls to be different within each study. Under this scenario we simulated case (c), with the numbers of samples (case/control) being 60/20, 60/20, 60/20 for three studies respectively. In scenario 3 , we allowed the ratios of case and control samples to be different within each study. Under this scenario we simulated case (d) with the numbers of samples (case/control) being 20/60, 40/40, 60/20 for three studies respectively.

The results of simulation cases (a)-(d) are shown in Supplementary Table S2 [Huo, Song and Tseng (2019)]. We observed that under scenario 1, scenario 2, 
and scenario 3, BayesMP controlled FDR to its nominal level for $\mathcal{D}_{\bar{A}}, \mathcal{D}_{B}$ and $\mathcal{D}_{\bar{r}}$. These results indicate that our Bayesian model is robust against impact of heterogeneity sample size in a wide spectrum of scenarios.

3.4. Additional simulations on robustness of the algorithm. In our Bayesian hierarchical model we assume that the null component $f_{0}$ comes from $\mathrm{N}(0,1)$ the theoretical null for all studies. However, this assumption can be violated when genes from null components are correlated [Efron et al. (2001)]. Therefore, we designed simulations to access the performance of our model when theoretical null assumption is not valid. To be specific, in our simulation setting step (c) we varied the number of correlated clusters $C=200,300,400,500$, representing increasing probability of correlated null component. We evaluated the performance of the Bayesian hierarchical model, and the result is shown in Supplementary Table S4 [Huo, Song and Tseng (2019)]. We observe that the BayesMP still performs very well even though the null components are correlated. Therefore BayesMP is robust against the theoretical null assumption for the null component.

4. Real data applications. To further evaluate our method and demonstrate its usage, we applied BayesMP on three real meta-analysis examples: one on the gene expression of multi-tissue microarray studies using metabolism-related knockout mice, one on multi-brain-region RNA-seq studies using HIV transgenic rats and another on transcriptomic breast cancer studies across multiple platforms. The sample size description is shown in Supplementary Table S5 [Huo, Song and Tseng (2019)].

4.1. Mouse metabolism data. Very long-chain acyl-CoA dehydrogenase (VLCAD) deficiency was found to be associated with energy metabolism disorder in children [Li and Tseng (2011)]. Two genotypes of the mouse model-wild type $(\mathrm{VLCAD}+/+)$ and VLCAD-deficient (VLCAD -/-) - were studied for three types of tissues (brown fat, liver and heart) with three to four mice in each genotype group. The total number of genes from these three transcriptomic microarray studies is 14,495. Supplementary Table S5(a) [Huo, Song and Tseng (2019)] shows details of the study design. Two-sided $p$-values were calculated using limma by comparing wild type versus VLCAD-deficient mice in each tissue, and one-sided $p$-values were obtained by considering the effect size direction. BayesMP took 62 minutes to obtain 10,000 posterior samples using MCMC, and the first 500 posterior samples were excluded as burn-in iterations. By controlling FDR at 5\%, we detected 168 probes under $\mathcal{D}_{\bar{A}}$; among them 156 have concordant effect size directions in all the three tissues. The heatmap for the genes detected under $\mathcal{D}_{\bar{A}}$ is shown in Supplementary Figure S6 [Huo, Song and Tseng (2019)].

Similarly, under $\mathcal{D}_{B}$ we obtained 3496 DE genes at an FDR level of 5\% and 1243 DE genes at an FDR level of $1 \%$. Due to the unusually strong genome-wide biological signal, we decided to use the FDR cutoff of $1 \%$ for the downstream 
analysis to increase statistical power (of the Fisher's exact test) in pathway enrichment analysis. Then we applied the tight clustering algorithm using the cosine distance as described in Section 2.4 to detect modules based on 1243 DE genes at the stringent FDR level of $1 \%$. The results are shown in Figure 3. Using the tight clustering, we were able to detect six gene modules with unique patterns. The first two biomarker modules are consensus genes that are up-regulated or down-regulated in all tissues. The next four modules are biomarkers with study-specific differential patterns. For example, DE genes in module III are up-regulated in the heart but not in the brown fat or the liver. To examine the biological functions of these modules, we performed pathway enrichment analysis for genes in each module using Fisher's exact test. The pathway database was downloaded from the Molecular Signatures Database (MSigDB) v5.0 (http://bioinf.wehi.edu.au/software/MSigDB/), where a mouse-version pathway database was created by combining pathways from KEGG, BIOCARTA, REACTOME and GO databases and mapping all the human genes to their orthologs in mouse using Jackson Laboratory Human and Mouse Orthology Report (http://www.informatics.jax.org/orthology.shtml). The resulting $p$-values were converted to $q$-values by Benjamini-Hochberg correction [Benjamini and Hochberg (1995)] to adjust for multiple comparison, where $q$ value measures the false discovery rate (FDR) one would incur by accepting the given test. At an FDR cutoff of 5\%, we summarized the pathway detection result (see Supplementary Excel file 1 for detailed pathway information). Among the six gene modules with distinct DE patterns, module I is enriched in enzyme pathways (e.g., KEGG lysosome; $q=1.6 \times 10^{-3}$ ), module II is enriched in pathways for lyase activity (e.g., GO lyase activity; $q=0.26$ ), module III is enriched in defense response pathways (e.g., GO defense response pathway; $q=2.2 \times 10^{-6}$ ), module IV is enriched in phosphatase regulator pathways (e.g., GO phosphatase regulator activity; $q=0.12$ ) and module VI is enriched in platelet related pathways (e.g., GO formation of platelet; $q=2.2 \times 10^{-2}$ ). For module V we didn't detect any enriched pathways. Remarkably, all of these pathways are known to be related to different aspects of metabolism which indicates that our method is able to detect homogeneous and heterogeneous gene modules that are biologically meaningful. The biomarker clustering result enhances meta-analysis interpretation and motivates hypothesis for further biological investigation. For example, it is intriguing to understand why VLCAD mutation impacts DE genes only up-regulated in the heart but not in the brown fat or the liver, and why these genes are associated with the defense response pathway.

4.2. HIV transgenic rat RNA-seq data. Li et al. (2013) conducted studies to determine gene expression differences between F344 and HIV transgenic rats using RNA-seq (GSE47474 in the Gene Expression Omnibus database [http:// www.ncbi.nlm.nih.gov/geo/query/acc.cgi?acc=GSE47474]). The HIV transgenic rat model was designed to study learning, memory, vulnerability to drug addiction and other psychiatric disorders vulnerable to HIV-positive patients. They 
I.

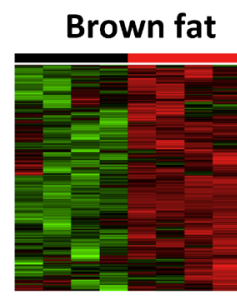

II.

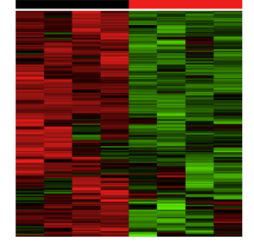

III.

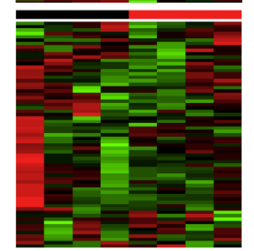

IV.

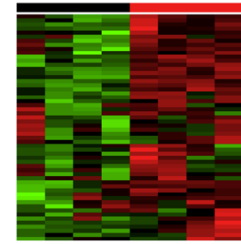

V.
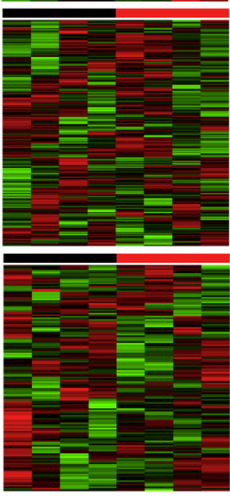
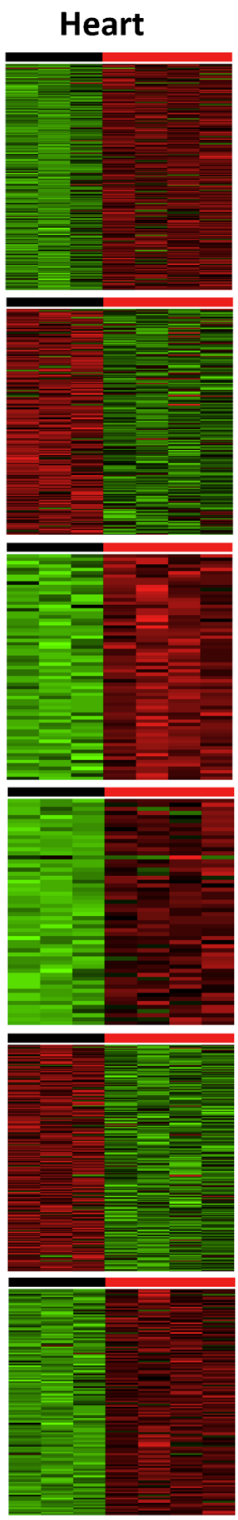

(a) Heatmap
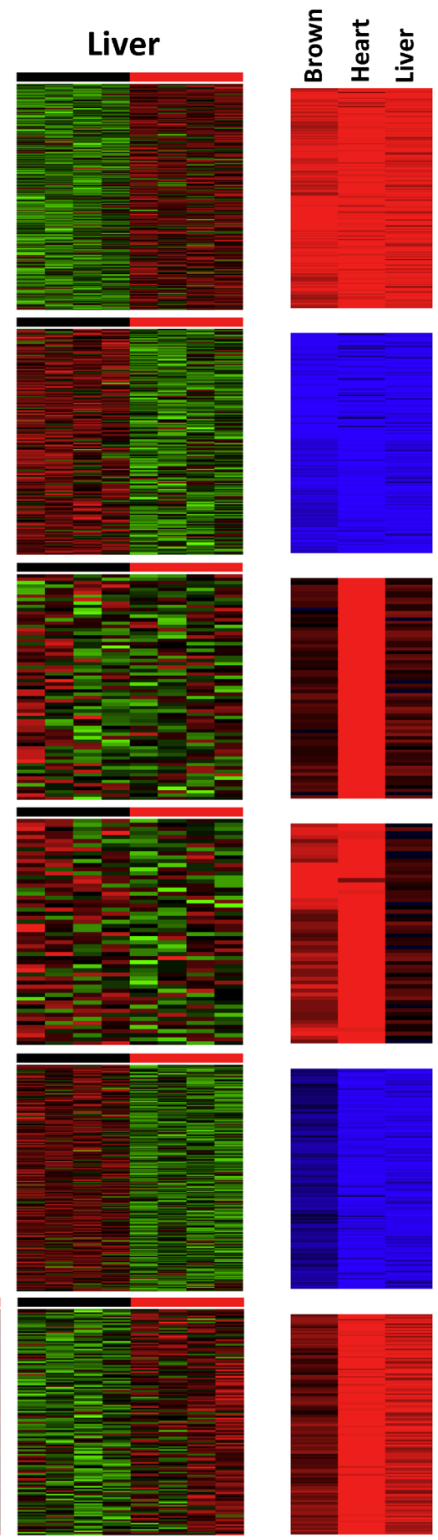

(b) CS

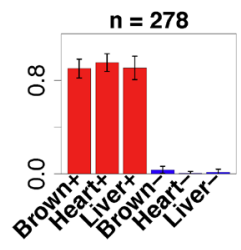

$\mathbf{n}=192$

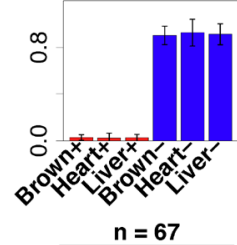

$\stackrel{\infty}{\circ}$

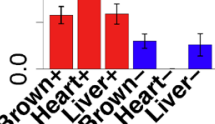

$\mathrm{n}=\mathbf{5 6}$

$\stackrel{\infty}{\circ}$

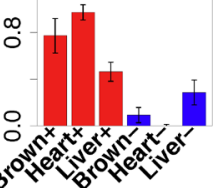

$\mathbf{n}=\mathbf{2 3 2}$

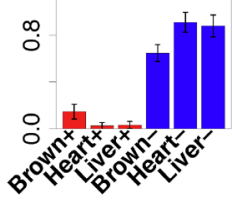

$n=142$

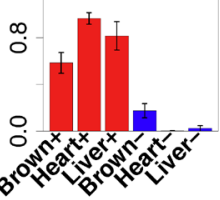

(c) bar plot

FIG. 3. Six meta-pattern modules of biomarkers from the mouse metabolism example. Each row shows a set of detected biomarkers showing similar meta-pattern of differential signals. (a) Heatmaps of detected genes (on the rows) and samples (on the columns) for each tissue (brown fat, heart, or liver), where each tissue represents a study (i.e., brown fat for $s=1$, heart for $s=2$, liver for $s=3$ ). The black color bar on top represents wild type (control), and the red color bar on top represents $V L C A D$-deficient mice (case). (b) Heatmaps of confidence scores (CS) (genes on the rows and three studies on the columns). Confidence score is described in Section 2.3 which ranges from -1 (blue 
sequenced RNA transcripts with 12 F334 rats and 12 HIV transgenic rats in prefrontal cortex (PFC), hippocampus (HIP) and striatum (STR) brain regions (see detail in Supplementary Table S5(b) [Huo, Song and Tseng (2019)]). We applied the same alignment procedure using TopHat [Trapnell, Pachter and Salzberg (2009)] adopted by Li et al. (2013) and obtained the RNA-seq count data for 16,821 genes by BEDTools [Quinlan and Hall (2010)]. We filtered out genes with less than 100 total counts within any brain region and ended up with 11,824 genes. We removed potential outliers by checking the sample correlation heatmaps (Supplementary Figure S7 [Huo, Song and Tseng (2019)]). We employed R package edgeR to perform $\mathrm{DE}$ gene detection and obtained two-sided $p$-values. The one-sided $p$-values were obtained by considering the effect size directions and further converted to $\mathrm{Z}$ statistics. It took 41 minutes to obtain 10,000 posterior samples via MCMC, and the first 500 posterior samples were excluded as burn-in iterations. Since it is well known that the postmortem brain expression profiles generally contain weak signals, we controlled FDR at $20 \%$ in the analysis. Under $\mathcal{D}_{\bar{A}}$ we detected 69 genes, of which all 69 had concordant DE directions. The heatmaps of the expression levels (log of normalized counts) of these genes in the three brain regions are shown in Supplementary Figure S8 [Huo, Song and Tseng (2019)]. Under $\mathcal{D}_{B}$ we detected 669 genes. We further applied the tight clustering algorithm, and obtained three gene modules. Their gene expression heatmaps, DE confidence scores and bar plots of posterior probability of differential expression are shown in Figure 1. To examine the biological functions of these modules, we also performed pathway enrichment analysis using the same procedure as in Section 4.1 (see Supplementary Excel file 2 for detailed information). As the postmortem brain expression profiles generally contain much weaker signals and the gene size of each module is relatively small, we presented $p$-values (unadjusted for multiple comparison) instead of $q$-values for the below pathway enrichment analysis. According to the results, module $I$ is down-regulated in all three brain regions and is enriched in pathways related to the immune system (e.g., REACTOME innate immunity signaling, $p=6.26 \times 10^{-3}$ ), module II is up-regulated in all three brain regions and is enriched in pathways related to response to virus (e.g., GO response to virus, $p=1.81 \times 10^{-3}$ ), module III is down-regulated in HIP but up-regulated in PFC and STR, and it is enriched in pathways related to synapsis (e.g., GO synaptic transmission, $p=2.75 \times 10^{-3}$ ) and neuron connections (e.g., KEGG neuroactive ligand receptor interaction, $\left.p=2.88 \times 10^{-3}\right)$. Since it is well known that HIV attacks the immune system [Weiss (1993)], we anticipate genes for immune response

FIG. 3 (Continued). color for down-regulation) to 1 (red color for up-regulation). (c) Bar plots of mean posterior probability for $Y_{g s}=1$ (red color for up-regulation) and $Y_{g s}=-1$ (blue color for down-regulation) for each module in each tissue. Error bar represents standard deviation across all genes in the module. The number of genes is shown on top of each bar plot. In panels (b) and (c), we use "Brown" to denote "Brown fat." 
to be down-regulated, as observed in module I. The up-regulation of response to virus pathway we found in module II is reasonable since the mice are infected by the virus. Moreover, because different brain regions have different functions, it is not surprising to discover some neuron-related genes that may respond differently to HIV in different brain regions (module III).

4.3. Breast cancer dataset. In this example we combined seven breast cancer transcriptomic datasets which study the same biological problem using different gene expression platforms, including Illuminia, Affymetrix and RNA-seq. The phenotype of interest is the breast cancer grade which is defined according to the cancer cells' growth patterns as well as their appearance compared to to healthy breast cells. Grade I cancer cells show slow and well-organized growth patterns, and they look a little bit different from healthy cells, while grade III cancer cells grow quickly in disorganized patterns with many dividing to make new cancer cells which look very different from healthy cells. Details of these seven datasets are described in Supplementary Table S5(c) [Huo, Song and Tseng (2019)]. For each study, if multiple probes match to the same gene, we select the probe with the largest IQR [Gentleman et al. (2006)] to represent the gene. After matching the same gene symbols, 3920 genes that appeared in all seven studies were selected for the analysis. For each study we obtained two-sided $p$-values using limma (for continuous data) or edgeR (for count data) by comparing grade I versus grade III with adjustment of race, age and gender as covariates whenever they were available. We calculated one-sided $p$-values by considering the direction of the effect sizes. We applied BayesMP; it took 31 minutes to obtain 10,000 posterior samples using MCMC, and the first 500 posterior samples were excluded as burn-in iterations.

Since we expect the studies combined in this application to be more homogeneous than previous examples, genes DE in most of the studies are our major interest and worth further investigation. Moreover, because the studies are conducted by different groups using different platforms, we want our analysis to be robust against a couple of studies with poor quality or unspecific probe design. We visualize the number of declared DE genes at FDR $5 \%$ for $\mathcal{D}_{\bar{r}}(r=1, \ldots, 7)$ in Figure 4. It is noticed that there is a noticeable drop in the number of DE genes between $r=6$ and $r=7$. This indicates that it could be too stringent to require that DE genes agree in all seven studies. To make the DE gene detection replicable enough yet not too stringent, we chose to use $\mathcal{D}_{\bar{r}}(r=6)$. Under FDR 5\%, we detected 1437 significant genes. Pathway enrichment analysis was performed using Fisher's exact test. Top pathways associated with these genes are REACTOME cell cycle $(q=0.0066)$ and REACTOME DNA replication $(q=0.0066)$. These results are biologically meaningful since it is known that cancer cells' growth patterns of different grades are associated with cell cycle and DNA replication.

We further performed a cross-study validation to assess the performance of BayesMP. In each iteration we set aside one study, applied BayesMP to the remaining six studies and declared DE genes under $\mathcal{D}_{\bar{A}}$ using FDR $=5 \%$. To evaluate the consistency of DE gene detection results between the BayesMP of the six 


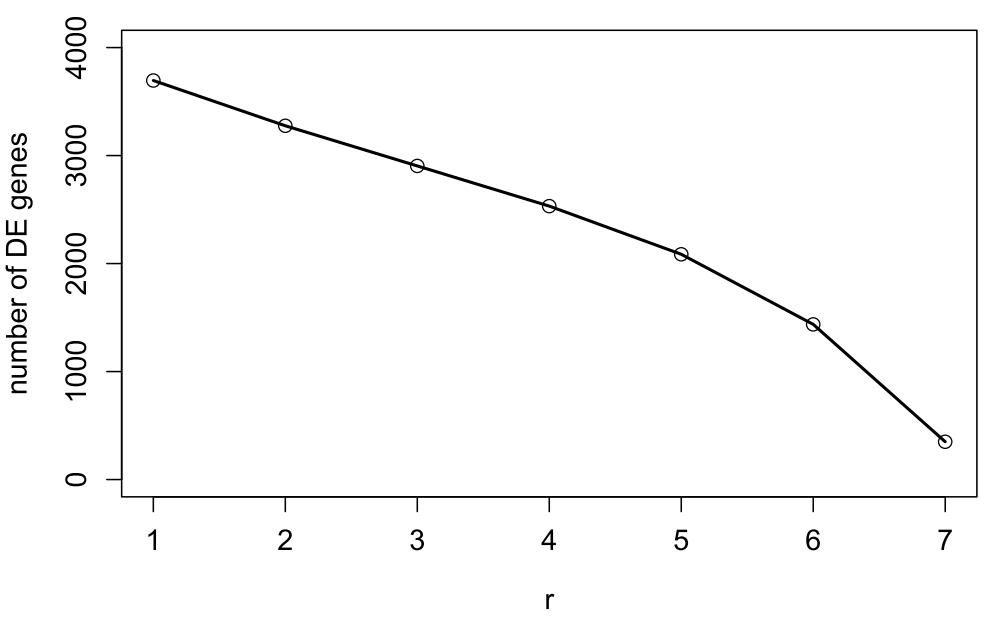

FIG. 4. Number of declared DE genes at FDR $5 \%$ for $\mathcal{D}_{\bar{r}}(r=1, \ldots, 7)$.

studies and the left-alone study, we calculated the area under the curve (AUC) of the receiver operating characteristic (ROC) curves by treating the DE status from BayesMP as a binary outcome and using the $p$-values from the left-alone study to determine the moving sensitivity and specificity. We repeated this procedure for each left-alone study to calculate the AUC values. As a baseline contrast, for each left-alone single study, we also similarly calculated AUC values using the DE status (using FDR $=5 \%$ ) from each of the other six individual studies, and calculated the average and standard error of AUCs. As shown in Supplementary Table 6 [Huo, Song and Tseng (2019)], the AUCs from BayesMP were between 0.65 and 0.85 , consistently higher than the average AUCs from six individual studies (ranging from 0.59 to 0.75 ) which shows quantitative validity of the meta-analysis. Study GSE6532 has a relatively lower cross-study validation AUC from BayesMP $(=0.65)$ than other studies, indicating lower compatibility with other studies. This also justifies the usage of $r=6$ for $\mathcal{D}_{\bar{r}}$ in the previous paragraph.

5. Conclusion. For meta-analysis at the genome-wide level, the issues to efficiently integrate information across studies and genes and to quantify homogeneous and heterogeneous DE signals across studies have brought new statistical challenges. The Bayesian hierarchical model provides a feasible and effective solution. Compared to traditional hypothesis testing, decision theory framework from Bayesian modeling provides a more flexible inference to determine DE genes from meta-analysis. In this paper we proposed a Bayesian hierarchical model for general transcriptomic meta-analysis. From posterior distribution of the latent variable (DE indicators $Y_{g s}$ ), FDR is well controlled, and there is no need to select different test statistics for different hypothesis settings $\left(\mathrm{HS}_{\bar{A}}, \mathrm{HS}_{B}\right.$ and $\mathrm{HS}_{\bar{r}}$ ). Post hoc clustering analysis on the detected biomarkers generates biomarker modules of 
different meta-patterns that facilitate biological interpretation and provides clues for hypothesis generation and biological investigation.

Our proposed BayesMP framework has the following advantages. First, the model is simple, yet practical and powerful. The model is based on one-sided $p$ values. This allows easy integration of data from different platforms (e.g., many different platforms from microarray and RNA-seq). As a contrast Scharpf et al. (2009) described a full Bayesian hierarchical model for microarray meta-analysis, where the input data are microarray raw data (normalized intensities). Although such a full Bayesian model theoretically best integrates all information and can be more powerful, it cannot combine new RNA-seq platforms, since RNA-seq generates count data versus continuous intensity measures in microarray. Such a full hierarchical model also runs a greater risk of model misspecification that increases systemic bias across different microarray platforms. Our framework, based on $p$ values, circumvents these difficulties and is powerful as long as the method used to generate $p$-values in each study is effective. Second, we adopted a conjugate Bayesian approach using DPs for alternative distributions $f_{ \pm 1}^{(s)}$ which enables a mixture of multiple subgroups instead of a single one-component alternative. DPs is nonparametric and thus robust against model assumptions. The conjugacy of our model guarantees the fast computing of the Gibbs sampling procedure. Third, we have shown that decision theory framework from BayesMP provides good FDR control and power under different hypothesis settings (or decision spaces). Fourth, in contrast to the "hard" decision of 0 or 1 weights in AW-Fisher, the posterior distributions of the DE indicators $Y_{g s}$ provide a stochastic quantification and "soft" decision. For example, in the mouse metabolism example, gene Mbnl2 (probeset 1422836_at) and gene Bcl2111 (probeset 1435449_at) have very similar p-values in the three studies: $(0.0063,0.16,0.097)$ for Mbnl2 and $(0.0070,0.16,0.098)$ for $\mathrm{Bcl} 2111$. Using $\mathrm{AW}, \mathrm{Mbnl} 2$ ended up with a $p$-value of 0.020 with weights $(1,0,1)$, but $\operatorname{Bcl} 2111$ had a $p$-value of 0.021 with weights $(1,1,1)$. A slight alteration of the $p$-value in the second study (heart tissue) resulted in different weights. The posterior probabilities for these two genes are, however, very similar, with $(0.827,0.561,0.671)$ and $(0.819,0.561,0.662)$ respectively, and they belonged to the same gene module I in Figure 3. The stochastic quantification avoids sensitive 0 or 1 weight changes in AW-Fisher. Finally, Fisher's method does not categorize DE genes with homogeneous or heterogeneous DE patterns across studies. In contrast the improved AW-Fisher method allows categorization of biomarkers but generates up to $2^{S}-1$ biomarker clusters which becomes intractable when $S$ is large. The posterior probability of $Y_{g s}$ from BayesMP allows the application of tight clustering to directly identify tight clusters of biomarkers with distinct DE meta-patterns. Our simulation and three applications have shown good clustering accuracy and improved interpretation of the biomarker modules.

BayesMP potentially has the following potential limitations. Computing is often a consideration for Bayesian approaches. Our experiences have shown that 10,000 
simulations are sufficient to generate the posterior probabilities in general, and less than one hour is enough for combining around 10,000 genes using a regular desktop. For applications to much larger numbers of features (e.g., SNPs or methylation sites in methyl-seq), parallel computing and/or faster MCMC techniques will be needed.

Efron (2004) recommended to estimate an empirical null distribution for the $Z$-statistics when the null distribution deviates from $\mathrm{N}(0,1)$. In our simulation we have shown that BayesMP with theoretical null generates robust results when genes from null are correlated, a scenario violating the theoretical null assumption. We also found that BayesMP with empirical null is slightly overconservative (actually FDR 1\% while nominal FDR 5\%) when noise level is large. In our R package we allow the users to choose from using the theoretical null or the empirical null. However, the user should check the assumptions made when estimating the empirical null distributions. For example, Efron (2004) assumes less than 10\% DE genes and that empirical null is also from a Gaussian distribution. This assumption needs to be examined post hoc (e.g., examine whether the DE proportion is less than $10 \%$ in each study given a relatively loose FDR control, say FDR $<10 \%$ ). To explore whether theoretical or empirical null is more appropriate, we also provide histograms for visual diagnosis (see Supplementary Figures S4 and S5 [Huo, Song and Tseng (2019)] for details).

As mentioned in the Introduction, batch effect correction and direct merging could be a viable alternative for meta-analysis if raw data are available and batch effects can be accurately identified and corrected. Two major types of batch effect correction methods have been widely studied in DE analysis. The first type considers known batch information (aka unwanted variation [UV] factors; e.g., experiments performed on different dates, by different technicians or on different platforms). Many methods have been developed to correct for these known UVs [e.g., Johnson, Li and Rabinovic (2007), Walker et al. (2008)], and then samples can be directly merged for so-called mega-analysis. The second type of batch correction assumes the existence of unknown UV factors, in which case many methods [e.g., Kang, Ye and Eskin (2008), Leek and Storey (2007), Listgarten et al. (2010)] have been developed to eliminate effects from unknown UV factors to improve DE analysis. Since BayesMP takes $p$-values from single studies as input, these methods can be easily adopted in each single study before implementing BayesMP. However, it should be noted that overcorrection can be a potential concern for any batch correction method, and one should use with caution [Jacob, Gagnon-Bartsch and Speed (2016)]. In addition such batch correction is unnecessary if each study is from a single batch and there are no other hidden factors within each study.

A prior for $\sigma_{c}^{2}$ can be given to better characterize the variability in $\sigma_{c}^{2}$ (e.g., truncated inverse gamma distribution; here, truncation such that $\sigma_{c}^{2} \geq 1$ is a sufficient condition such that density function of $Z$-statistics is monotone with respect to $Z$ ). 
However, such a prior will make the Bayesian procedure lose conjugacy. Therefore, we fix $\sigma_{c}^{2}=1$ to keep the algorithm computationally efficient. Ghosal, Ghosh and Ramamoorthi (1999) illustrated that this procedure is equivalent to choosing the bandwidth parameter a priori in kernel density estimation, and established posterior consistency for it.

BayesMP is implemented in $\mathrm{R}$ calling $\mathrm{C}++$. The BayesMP package is publicly available at GitHub https://github.com/Caleb-Huo/BayesMP and the authors' websites.

Acknowledgments. The authors sincerely thank the Editor, the Associate Editor and the reviewers for their constructive comments which helped us improve the quality of this paper. We also thank Ohio Supercomputer Center (1987) for the computational resource.

\section{SUPPLEMENTARY MATERIAL}

Supplementary information (DOI: 10.1214/18-AOAS1188SUPPA; .pdf). Additional tables, figures, and text.

Supplementary Excel file 1 (DOI: 10.1214/18-AOAS1188SUPPB; .zip). Pathway information for the mouse metabolism application.

Supplementary Excel file 2 (DOI: 10.1214/18-AOAS1188SUPPC; .zip). Pathway information for the HIV transgenic rat application.

\section{REFERENCES}

Anders, S. and Huber, W. (2010). Differential expression analysis for sequence count data. Genome Biol. 11 Art. ID R106.

BenJAmini, Y. and HelLER, R. (2008). Screening for partial conjunction hypotheses. Biometrics 64 1215-1222. MR2522270

Benjamini, Y. and Hochberg, Y. (1995). Controlling the false discovery rate: A practical and powerful approach to multiple testing. J. Roy. Statist. Soc. Ser. B 57 289-300. MR1325392

Berger, J. O. (2013). Statistical Decision Theory and Bayesian Analysis, Springer, New York. MR0804611

Bhattacharjee, S., Rajaraman, P., Jacobs, K. B., Wheeler, W. A., Melin, B. S., Hartge, P., Yeager, M., Chung, C. C., Chanock, S. J., Chatterjee, N. et al. (2012). A subset-based approach improves power and interpretation for the combined analysis of genetic association studies of heterogeneous traits. Am. J. Hum. Genet. 90 821-835.

Birnbaum, A. (1954). Combining independent tests of significance. J. Amer. Statist. Assoc. 49 559-574. MR0065101

Chang, L.-C., Lin, H.-M., Sibille, E. and Tseng, G. C. (2013). Meta-analysis methods for combining multiple expression profiles: Comparisons, statistical characterization and an application guideline. BMC Bioinform. 14 Art. ID 368.

Cooper, H., Hedges, L. V. and Valentine, J. C. (2009). The Handbook of Research Synthesis and Meta-Analysis. Russell Sage Foundation, New York. 
DOMANY, E. (2014). Using high-throughput transcriptomic data for prognosis: A critical overview and perspectives. Cancer Res. 74 4612-4621.

EFron, B. (2004). Large-scale simultaneous hypothesis testing: The choice of a null hypothesis. J. Amer. Statist. Assoc. 99 96-104. MR2054289

Efron, B. (2008). Microarrays, empirical Bayes and the two-groups model. Statist. Sci. 23 1-22. MR2431866

Efron, B. (2009). Empirical Bayes estimates for large-scale prediction problems. J. Amer. Statist. Assoc. 104 1015-1028. MR2562003

EFron, B. and TiBShiRANi, R. (2002). Empirical Bayes methods and false discovery rates for microarrays. Genet. Epidemiol. 23 70-86.

Efron, B., Tibshirani, R., Storey, J. D. and Tusher, V. (2001). Empirical Bayes analysis of a microarray experiment. J. Amer. Statist. Assoc. 96 1151-1160. MR1946571

ESCOBAR, M. D. and WEST, M. (1995). Bayesian density estimation and inference using mixtures. J. Amer. Statist. Assoc. 90 577-588. MR1340510

FISHER, R. A. (1934). Statistical Methods for Research Workers. Hafner Publishing Co., New York. MR0346954

Gentleman, R., Carey, V. J., Huber, W., Irizarry, R. A. and Dudoit, S., eds. (2006). Bioinformatics and Computational Biology Solutions Using R and Bioconductor. Springer, New York. MR2201836

Ghosal, S., Ghosh, J. K. and Ramamoorthi, R. V. (1999). Posterior consistency of Dirichlet mixtures in density estimation. Ann. Statist. 27 143-158. MR1701105

Huo, Z., Ding, Y., Liu, S., Oesterreich, S. and Tseng, G. (2016). Meta-analytic framework for sparse $K$-means to identify disease subtypes in multiple transcriptomic studies. J. Amer. Statist. Assoc. 111 27-42. MR3494636

Huo, Z., Song, C. and Tseng, G. (2019). Supplement to "Bayesian latent hierarchical model for transcriptomic meta-analysis to detect biomarkers with clustered meta-patterns of differential expression signals." DOI:10.1214/18-AOAS1188SUPPA, DOI:10.1214/18-AOAS1188SUPPB, DOI:10.1214/18-AOAS1188SUPPC.

JACOB, L., GAGNON-BARTSCH, J. A. and SPEed, T. P. (2016). Correcting gene expression data when neither the unwanted variation nor the factor of interest are observed. Biostatistics 17 16-28. MR3449847

Johnson, W. E., Li, C. and Rabinovic, A. (2007). Adjusting batch effects in microarray expression data using empirical Bayes methods. Biostatistics 8 118-127.

KANG, H. M., YE, C. and EsKIN, E. (2008). Accurate discovery of expression quantitative trait loci under confounding from spurious and genuine regulatory hotspots. Genetics 180 1909-1925.

LEeK, J. T. and StOREY, J. D. (2007). Capturing heterogeneity in gene expression studies by surrogate variable analysis. PLoS Genet. 3 Art. ID e161.

LI, J. and TSENG, G. C. (2011). An adaptively weighted statistic for detecting differential gene expression when combining multiple transcriptomic studies. Ann. Appl. Stat. 5 994-1019. MR2840184

Li, M. D., Cao, J., Wang, S., Wang, J., Sarkar, S., Vigorito, M., Ma, J. Z. and ChANG, S. L. (2013). Transcriptome sequencing of gene expression in the brain of the HIV1 transgenic rat. PLOS ONE 8 Art. ID e59582.

LI, Q., WAnG, S., HuAnG, C.-C., Yu, M. and ShaO, J. (2014). Meta-analysis based variable selection for gene expression data. Biometrics 70 872-880. MR3295748

Listgarten, J., Kadie, C., Schadt, E. E. and Heckerman, D. (2010). Correction for hidden confounders in the genetic analysis of gene expression. Proc. Natl. Acad. Sci. USA 107 1646516470.

LitTELl, R. C. and FolKs, J. L. (1971). Asymptotic optimality of Fisher's method of combining independent tests. J. Amer. Statist. Assoc. 66 802-806. MR0312634 
MÜLler, P. and QuintanA, F. A. (2004). Nonparametric Bayesian data analysis. Statist. Sci. 19 95-110. MR2082149

Muralidharan, O. (2010). An empirical Bayes mixture method for effect size and false discovery rate estimation. Ann. Appl. Stat. 4 422-438. MR2758178

NeAL, R. M. (2000). Markov chain sampling methods for Dirichlet process mixture models. J. Comput. Graph. Statist. 9 249-265. MR1823804

Newton, M. A., Noueiry, A., SARKar, D. and Ahlquist, P. (2004). Detecting differential gene expression with a semiparametric hierarchical mixture method. Biostatistics 5 155-176.

OHIO SuPERCOMPUter Center (1987). Ohio Supercomputer Center. Available at http://osc.edu/ ark:/19495/f5s1ph73.

Quinlan, A. R. and HALL, I. M. (2010). BEDTools: A flexible suite of utilities for comparing genomic features. Bioinformatics 26 841-842.

Ramasamy, A., Mondry, A., Holmes, C. C. and Altman, D. G. (2008). Key issues in conducting a meta-analysis of gene expression microarray datasets. PLoS Med. 5 Art. ID e184.

Robinson, M. D., MCCARThy, D. J. and SMYTH, G. K. (2010). edgeR: A Bioconductor package for differential expression analysis of digital gene expression data. Bioinformatics 26 139-140.

Scharpf, R. B., TJelmeland, H., Parmigiani, G. and Nobel, A. B. (2009). A Bayesian model for cross-study differential gene expression. J. Amer. Statist. Assoc. 104 1295-1310. MR2750563

Simon, R. (2005). Development and validation of therapeutically relevant multi-gene biomarker classifiers. J. Natl. Cancer Inst. 97 866-867.

Simon, R., Radmacher, M. D., Dobbin, K. and McShane, L. M. (2003). Pitfalls in the use of DNA microarray data for diagnostic and prognostic classification. J. Natl. Cancer Inst. 95 14-18.

SмYтH, G. K. (2005). Limma: Linear models for microarray data. In Bioinformatics and Computational Biology Solutions Using R and Bioconductor 397-420. Springer, New York.

Song, C. and TsEnG, G. C. (2014). Hypothesis setting and order statistic for robust genomic metaanalysis. Ann. Appl. Stat. 8 777-800. MR3262534

Stouffer, S. A., Suchman, E. A., Devinney, L. C., Star, S. A. and Williams JR., R. M. (1949). The American Soldier: Adjustment During Army Life. Princeton Univ. Press, Princeton, NJ.

Trapnell, C., Pachter, L. and Salzberg, S. L. (2009). TopHat: Discovering splice junctions with RNA-Seq. Bioinformatics 25 1105-1111.

Tseng, G. C., Ghosh, D. and Feingold, E. (2012). Comprehensive literature review and statistical considerations for microarray meta-analysis. Nucleic Acids Res. 40 3785-3799.

Tseng, G. C. and Wong, W. H. (2005). Tight clustering: A resampling-based approach for identifying stable and tight patterns in data. Biometrics 61 10-16. MR2129196

Tusher, V. G., TibshiRANI, R. and ChU, G. (2001). Significance analysis of microarrays applied to the ionizing radiation response. Proc. Natl. Acad. Sci. USA 985116.

Walker, W. L., Liao, I. H., Gilbert, D. L., Wong, B., Pollard, K. S., McCulloch, C. E., LIT, L. and SHARP, F. R. (2008). Empirical Bayes accomodation of batch-effects in microarray data using identical replicate reference samples: Application to RNA expression profiling of blood from Duchenne muscular dystrophy patients. BMC Genomics 9 Art. ID 494.

WeIsS, R. A. (1993). How does HIV cause AIDS? Science 260 1273-1279.

ZhAO, Y., KANG, J. and YU, T. (2014). A Bayesian nonparametric mixture model for selecting genes and gene subnetworks. Ann. Appl. Stat. 8 999-1021. MR3262543 
Z. HuO

DEPARTMENT OF BIOSTATISTICS

UNIVERSITY OF FLORIDA

GAINESVILLE, FLORIDA 32611

USA

E-MAIL: zhuo@ufl.edu
S. CHI

Division OF BIOSTATISTICS

College of Public Health

The OHIO State University

COlumbus, OHIO 43210

USA

E-MAIL: song.1188@osu.edu

G. TSENG

DEPARTMENT OF BIOSTATISTICS,

HUMAN GENETICS

AND COMPUTATIONAL BIOLOGY

UNIVERSITY OF PITTSBURGH

PitTsburgh, Pennnsylvania 15261

USA

E-MAIL: ctseng@pitt.edu 